\title{
Prevalence and Factors Associated With Caesarean Section in Rwanda: a Trend Analysis of Rwanda Demographic and Health Survey 2000 to 2019-20
}

Peter M. Kibe ( $\square$ kybkibe@gmail.com )

African Population and Health Research Center

Grace Wambura Mbuthia

Department of Community Health, Jomo Kenyatta University of Agriculture and Technology

Duncan Shikuku

Liverpool School of Tropical Medicine

\section{Catherine Akoth}

Institute of Tropical and Infectious Diseases, University of Nairobi, Kenya

\section{James Oguta}

School of Health and Related Research, University of Sheffield, United Kingdom

\section{Loise Ng'ang'a}

Partners in Health

\section{Samwel Maina Gatimu}

Department of Economics, Population and Development Studies, University of Nairobi, Kenya

\section{Research Article}

Keywords: Rwanda, caesarean section, women, maternal health, maternal mortality, Eastern Africa

Posted Date: October 28th, 2021

DOl: https://doi.org/10.21203/rs.3.rs-996942/v1

License: (c) This work is licensed under a Creative Commons Attribution 4.0 International License. Read Full License 


\section{Abstract}

Background: Caesarean section (CS) is an important medical intervention for reducing the risk of poor perinatal outcomes. However, CS trends in sub-Saharan Africa (SSA) continue to increase yet maternal and neonatal mortality and morbidity remain high. Rwanda, like many other countries in SSA, has shown an increasing trend in the use of CS. This study assessed the trends and factors associated with CS delivery in Rwanda over the past two decades.

Methods: We used nationally representative child datasets from the Rwanda Demographic and Health Survey 2000 to 2019-20. All births in the preceding three years to the survey were assessed for the mode of delivery. The participants' characteristics, trends and the prevalence of CS were analysed using frequencies and percentages. Unadjusted and adjusted logistic regression analyses were used to assess the factors associated with population and hospital-based CS in Rwanda for each of the surveys.

Results: The population-based rate of CS in Rwanda significantly increased from $2.2 \%(95 \% \mathrm{Cl} 1.8-2.6)$ in 2000 to $15.6 \%(95 \% \mathrm{Cl} 13.9-16.5)$ in 2019-20. Despite increasing in all health facilities over time, there was an almost four-fold difference in the rate of CS between private $(60.6 \%)$ and public health facilities $(15.4 \%)$ in 2019-20. The rates and odds of CS were disproportionately high among women of high socioeconomic groups, those who resided in Kigali city, had multiple pregnancies, and attended at least four antenatal care visits while the odds of CS were significantly lower among multiparous women and those who had female babies.

Conclusion: Over the past two decades, the rate of CS use in Rwanda increased significantly at health facility and population level with high regional and socio-economic disparities. There is a need to examine the disparities in CS trends and developing tailored policy guidelines to ensure proper use of CS in Rwanda.

\section{Background}

The burden for maternal and neonatal mortality remains disproportionately high in many low- and middleincome countries (LMICs) [1, 2]. About 99\% of all global maternal deaths occurred in LMICs between 1990 and 2010 with an estimated two-thirds occurring in sub-Saharan Africa (SSA) [1, 2]. Despite the high maternal deaths in SSA, Rwanda recorded a 79\% decline in maternal mortality ratio between 2000 and 2017 [1]. This reduction has been accelerated by increased access and use of skilled and competent care by women during the pregnancy continuum. However, about $73 \%$ of the global maternal deaths remain due to direct obstetric causes including obstetric interventions and complications [3] despite most of them being preventable. The provision of basic and comprehensive emergency obstetric and newborn care (EmONC) within a continuum of care is essential to reduce maternal and newborn morbidity and mortality $[4,5]$.

Caesarean section (CS) is an important comprehensive EmONC signal function and medical intervention for reducing risks of perinatal morbidity and mortality [6]. The proportion of delivery by CS is used to estimate the level of access and use of the intervention in saving maternal and child health at a population level [7]. Over the past few decades, global trends have shown a steady increase in CS use [8]. Gibbons and colleagues found that half of the countries surveyed globally had CS rates of above 15\% [9] while Betran and colleagues estimated that by $2030,29 \%$ of women will be giving birth through CS globally; about $7 \%$ in SSA and $63 \%$ in Eastern Asia [10]. Countries in SSA are also reporting high CS rates despite the high burden of preventable 
maternal and neonatal mortality [1]. However, huge disparities in CS use exist in SSA countries with some countries like South Sudan reporting rates lower than 1\% while others like Ghana reporting a prevalence of $16 \%$ $[11,12]$.

These observed global increases in CS are driven majorly by medical and non-medical factors [6, 9]. Medically, CS deliveries are indicated in high risk conditions such as placenta previa, breech presentation, contracted pelvis, post-term pregnancy and hypertensive disorders [13]. However, CS deliveries have been conducted due to non-medical factors including maternal age, socioeconomic status, literacy levels, occupation, religion and culture $[12,14]$ and other demand-driven factors such as private practice and the cost of accessing CS [12]. In LMICs, the causes for the increasing CS use remain unclear though socioeconomic factors have been shown to contribute to the increase $[6,15]$. The World Health Organization (WHO) recommends an optimal CS rate of 10$15 \%$ where rates below the lower limit suggest the unmet need for CS while rates above the upper limit suggest overuse of CS $[16,17]$. The use of CS without medical indication is of no significance in reducing maternal and child mortality [6, 7], with recent evidence showing no beneficial effect of CS rates of above $10 \%$ on perinatal mortality rate [7].

Although Rwanda has made great strides in reducing maternal and child mortality in recent years, there is a risk of reversing these gains through the overuse of CS. Rwanda, like many countries in SSA, has shown a consistent rise in the use of CS, which is estimated to account for $13 \%$ [18] to $25.9 \%$ [19] of all deliveries. A recent study reported CS rates of $64 \%$ in private health facilities in Rwanda [11]. In addition, evidence indicates that if uncontrolled, the use of CS will continue to rise and with it the dire consequences [20]. Therefore, understanding the trends and patterns of CS use is the mainstay of preventing risks and developing contextspecific evidence for policies to reduce maternal and neonatal mortality. However, comprehensive evidence on trends and patterns of CS use is lacking in Rwanda. This study used national-level data from the Rwanda Demographic and Health Survey between 2000 and 2019-20 to assess the trends and factors associated with CS delivery in Rwanda at both population and hospital levels.

\section{Methods}

\section{Study setting}

Rwanda is a low-income, agricultural and landlocked country with approximately 11 million people living in five regions covering an area of 26,338 square kilometres [20]. It has an average of 4.4 persons per household [19] and a gross domestic product per capita of US $\$ 780.80$ [21]. About half (48\%) of its population is under 19 years of age and $39 \%$ live below the poverty line with a life expectancy at birth of 71.1 years for women and an adult literacy rate of $80 \%$ among $15-49$ years old women. In addition, $87.3 \%$ of the population has health insurance and access to health services; spending an average of 47.4 minutes to reach a health centre [19]. In 2016, CS in Rwanda were conducted in 27 (75\%) of the 36 districts, provincial and referral hospitals [19].

\section{Data source and sample}

The study used the child datasets from the Rwanda Demographic Health Surveys (RDHS) conducted in 2000, 2005, 2010, 2014-15 and 2019-20 using stratified, two-stage cluster sampling [20, 22-25]. Households were 
stratified into urban or rural and all eligible women 15-49 years in selected households were interviewed using standard DHS questionnaires. All babies born within the preceding three years of each survey and with complete data were included in the population-based analysis while only babies delivered at a hospital were included in the hospital-based analysis. Of the 75,777 children born within the three years preceding each survey, 34,144 children were included after excluding 41,633 children with missing observations in the outcome variable (Suppl. Figure 1).

\section{Measures}

The outcome variable was delivery by CS, which was categorized into "Yes" or "No". Women were asked if they had been delivered by CS within the three years preceding the survey. Since it is possible for women to have more than one CS in three years, we used the participants' unique identifiers and weighted samples to account for the clustering of CS. The explanatory variables included in the study were identified from a review of literature on factors associated with CS use [12, 26-32]. Supplementary Table 1 operationalises these variables that include residence (urban or rural), maternal age at birth ( $<20,20-34$ and $\geq 35$ years), education status (no formal, primary and secondary or higher), access to information (Yes or No), marital status (in-a-union and notin-a-union), occupation (not working, agricultural and formal employment), place of delivery (private, public and home/others), antenatal care (ANC) attendance ( $<4$ and $\geq 4$ visits and missing), the weight of the baby (normal, low birth weight and big baby), sex of the baby (male or female), type of pregnancy (singleton or multiple), parity $(1,2-4, \geq 5)$, partner's education (no formal, primary and secondary or higher), and region (East, West, South, North and Kigali City).

\section{Statistical analysis}

We performed analyses for each of the surveys and the pooled datasets using Stata version 17.0 (Stata Corporation, College Station, TX). Pooled datasets were created by appending individual datasets for each of the five surveys. Participants' characteristics and trends in the prevalence of CS were analysed using frequencies and percentages. To assess the factors associated with population-based and hospital-based CS, bivariate and multivariable logistic regression models were performed for each of the surveys and pooled data. Variance inflation factor was used to assess multicollinearity. All variables included in the unadjusted model were hierarchically included in the adjusted model due to their importance in explaining CS. We reported both the unadjusted and adjusted odds ratios and considered significance at a p-value of less than or equal to 0.05 . Only children with complete data were included in the analyses. The reporting in this study were guided by the STROBE guidelines for cross-sectional studies (Suppl. Table 3) [33].

\section{Ethics}

The study used anonymised open-access secondary data from the RDHS, which received ethical approvals from the Rwanda National Ethics Committee and the Institutional Review Board of ICF International. The data were accessed upon approval of data request to the DHS program and were used as per the data agreement. Additional information on the ethical approvals and processes for the surveys can be obtained from the published reports [20, 22-25]. 


\section{Results}

\section{Respondents' characteristics}

Overall, the study included 34,144 children born within the three years preceding each survey (Suppl. Figure 1). A majority of the children were born to middle-aged women (20-34 years), women who resided in rural areas, had a primary level of education, had two to four children, and had attended less than four ANC visits across the years. An almost equal proportion of participants lived in the West, East, and South provinces of Rwanda with the smallest proportion of participants living in Kigali City (Table 1). 
Table 1

Participants' characteristics

\begin{tabular}{|c|c|c|c|c|c|c|c|c|c|c|}
\hline \multirow[t]{2}{*}{ Characteristics } & \multicolumn{2}{|c|}{$\begin{array}{l}2000 \\
(\mathrm{~N}=6706)\end{array}$} & \multicolumn{2}{|c|}{$\begin{array}{l}2005 \\
(\mathrm{~N}=7712)\end{array}$} & \multicolumn{2}{|c|}{$\begin{array}{l}2010 \\
(\mathrm{~N}=7159)\end{array}$} & \multicolumn{2}{|c|}{$\begin{array}{l}2014-15 \\
(\mathrm{~N}=6248)\end{array}$} & \multicolumn{2}{|c|}{$\begin{array}{l}2019-20 \\
(\mathrm{~N}=6319)\end{array}$} \\
\hline & $\mathrm{n}$ & $\%$ & $\mathrm{n}$ & $\%$ & $\mathbf{n}$ & $\%$ & $\mathrm{n}$ & $\%$ & $\mathrm{n}$ & $\%$ \\
\hline \multicolumn{11}{|l|}{ Age, years } \\
\hline $15-19$ & 431 & 6.4 & 318 & 4.1 & 342 & 4.8 & 344 & 5.5 & 286 & 4.5 \\
\hline $20-34$ & 4,881 & 72.8 & 5,830 & 75.6 & 5,605 & 78.3 & 4,875 & 78.0 & 4,520 & 71.5 \\
\hline $35-49$ & 1,394 & 20.8 & 1,564 & 20.3 & 1,212 & 16.9 & 1,029 & 16.5 & 1,513 & 23.9 \\
\hline \multicolumn{11}{|l|}{ Residence } \\
\hline Urban & 1,460 & 21.8 & 1,478 & 19.2 & 906 & 12.7 & 1,384 & 22.2 & 1,284 & 20.3 \\
\hline Rural & 5,246 & 78.2 & 6,234 & 80.8 & 6,253 & 87.3 & 4,864 & 77.9 & 5,035 & 79.7 \\
\hline \multicolumn{11}{|l|}{ Marital status } \\
\hline Not in a union & 841 & 12.5 & 904 & 11.7 & 988 & 13.8 & 1032 & 16.5 & 1,004 & 15.9 \\
\hline Married/cohabiting & 5,865 & 87.5 & 6,808 & 88.3 & 6,171 & 86.2 & 5,216 & 83.5 & 5,315 & 84.1 \\
\hline \multicolumn{11}{|l|}{ Education } \\
\hline No formal & 2,115 & 31.5 & 2,082 & 27.0 & 1,327 & 18.5 & 893 & 14.3 & 691 & 10.9 \\
\hline Primary & 3,802 & 56.7 & 4,941 & 64.1 & 5,222 & 72.9 & 4,479 & 71.7 & 4,076 & 64.5 \\
\hline $\begin{array}{l}\text { Secondary and } \\
\text { higher }\end{array}$ & 789 & 11.8 & 689 & 8.9 & 610 & 8.5 & 876 & 14.0 & 1,552 & 24.6 \\
\hline \multicolumn{11}{|l|}{ Occupation } \\
\hline Not working & 149 & 2.6 & 1,552 & 20.1 & 831 & 11.6 & 534 & 8.6 & 1,269 & 20.1 \\
\hline Agriculture & 4,915 & 86.4 & 5,475 & 71.0 & 5,473 & 76.5 & 4,572 & 73.2 & 2,510 & 39.7 \\
\hline $\begin{array}{l}\text { Formal } \\
\text { employment }\end{array}$ & 624 & 11.0 & 685 & 8.9 & 855 & 11.9 & 1,140 & 18.3 & 2,540 & 40.2 \\
\hline \multicolumn{11}{|l|}{ Wealth, quintiles } \\
\hline Poorest & 1,191 & 18.0 & 1,604 & 20.8 & 1,703 & 23.8 & 1,542 & 24.7 & 1,604 & 25.4 \\
\hline Poorer & 1,587 & 24.0 & 1,518 & 19.7 & 1,567 & 21.9 & 1,294 & 20.7 & 1,241 & 19.6 \\
\hline Average & 824 & 12.5 & 1,517 & 19.7 & 1,419 & 19.8 & 1,152 & 18.4 & 1,178 & 18.6 \\
\hline Richer & 1,300 & 19.7 & 1,591 & 20.6 & 1,289 & 18.0 & 1,067 & 17.1 & 1,142 & 18.1 \\
\hline Richest & 1,707 & 25.8 & 1,482 & 19.2 & 1,181 & 16.5 & 1,193 & 19.1 & 1,154 & 18.3 \\
\hline
\end{tabular}




\begin{tabular}{|c|c|c|c|c|c|c|c|c|c|c|}
\hline \multirow[t]{2}{*}{ Characteristics } & \multicolumn{2}{|c|}{$\begin{array}{l}2000 \\
(\mathrm{~N}=6706)\end{array}$} & \multicolumn{2}{|c|}{$\begin{array}{l}2005 \\
(\mathrm{~N}=7712)\end{array}$} & \multicolumn{2}{|c|}{$\begin{array}{l}2010 \\
(\mathrm{~N}=7159)\end{array}$} & \multicolumn{2}{|c|}{$\begin{array}{l}2014-15 \\
(\mathrm{~N}=6248)\end{array}$} & \multicolumn{2}{|c|}{$\begin{array}{l}2019-20 \\
(\mathrm{~N}=6319)\end{array}$} \\
\hline & $\mathrm{n}$ & $\%$ & $n$ & $\%$ & $\mathrm{n}$ & $\%$ & $\mathrm{n}$ & $\%$ & $n$ & $\%$ \\
\hline$<4$ & 3,452 & 51.5 & 50.85 & 50.0 & 2,929 & 40.9 & 39.36 & 38.1 & 2,367 & 37.5 \\
\hline$\geq 4$ & 442 & 6.6 & 7.659 & 7.0 & 1,668 & 23.3 & 31.33 & 29.9 & 2,102 & 33.3 \\
\hline Missing & 2,812 & 41.9 & 41.5 & 40.8 & 2,562 & 35.8 & 29.31 & 28.4 & 1,850 & 29.3 \\
\hline \multicolumn{11}{|l|}{ Parity } \\
\hline 1 & 694 & 10.4 & 786 & 10.2 & 1,173 & 16.4 & 1,222 & 19.6 & 1,141 & 18.1 \\
\hline $2-4$ & 3,505 & 52.3 & 3,925 & 50.9 & 3,776 & 52.7 & 3,617 & 57.9 & 3,612 & 57.2 \\
\hline $5+$ & 2,507 & 37.4 & 3,001 & 38.9 & 2,210 & 30.9 & 1,409 & 22.6 & 1,566 & 24.8 \\
\hline \multicolumn{11}{|l|}{ Baby's sex } \\
\hline Male & 3,415 & 50.9 & 3,923 & 50.9 & 3,601 & 50.3 & 3,149 & 50.4 & 3,205 & 50.7 \\
\hline Female & 3,291 & 49.1 & 3,789 & 49.1 & 3,558 & 49.7 & 3,099 & 49.6 & 3,114 & 49.3 \\
\hline \multicolumn{11}{|c|}{ Baby's birth weight } \\
\hline Average & 1,591 & 23.8 & 1,855 & 24.1 & 3,844 & 53.7 & 4581 & 73.4 & 4,839 & 76.6 \\
\hline Low birth weight & 218 & 3.3 & 207 & 2.7 & 501 & 7.0 & 600 & 9.6 & 692 & 11.0 \\
\hline Big baby & 4,872 & 72.9 & 5,650 & 73.3 & 2,814 & 39.3 & 1,061 & 17.0 & 788 & 12.5 \\
\hline \multicolumn{11}{|l|}{ Twin Status } \\
\hline Singleton & 6,542 & 97.6 & 7,518 & 97.5 & 6959 & 97.2 & 6069 & 97.1 & 6,151 & 97.3 \\
\hline Multiple & 164 & 2.5 & 194 & 2.5 & 200 & 2.8 & 179 & 2.9 & 168 & 2.7 \\
\hline \multicolumn{11}{|l|}{$\begin{array}{l}\text { Access to } \\
\text { information }\end{array}$} \\
\hline No & 2,375 & 35.4 & 1,661 & 21.5 & 673 & 9.4 & 1,028 & 16.5 & 1,359 & 21.5 \\
\hline Yes & 4,331 & 64.6 & 6,051 & 78.5 & 6,486 & 90.6 & 5,220 & 83.6 & 4,960 & 78.5 \\
\hline \multicolumn{11}{|c|}{ Partner's education } \\
\hline No formal & 1,916 & 29.8 & 1,986 & 27.1 & 1,318 & 19.7 & 906 & 15.9 & 673 & 12.7 \\
\hline Primary & 3,551 & 55.2 & 4,409 & 60.1 & 4,649 & 69.6 & 4,049 & 71.2 & 3,521 & 66.5 \\
\hline $\begin{array}{l}\text { Secondary and } \\
\text { higher }\end{array}$ & 961 & 15.0 & 945 & 12.9 & 715 & 10.7 & 730 & 12.8 & 1,103 & 20.8 \\
\hline \multicolumn{11}{|l|}{ Region } \\
\hline Kigali City & 837 & 12.5 & 504 & 6.5 & 750 & 10.5 & 729 & 11.7 & 731 & 11.6 \\
\hline West & 1,605 & 23.9 & 1,906 & 24.7 & 1,805 & 25.2 & 1,624 & 26.0 & 1,681 & 26.6 \\
\hline
\end{tabular}




\begin{tabular}{|c|c|c|c|c|c|c|c|c|c|c|}
\hline \multirow[t]{2}{*}{ Characteristics } & \multicolumn{2}{|c|}{$\begin{array}{l}2000 \\
(\mathrm{~N}=6706)\end{array}$} & \multicolumn{2}{|c|}{$\begin{array}{l}2005 \\
(\mathrm{~N}=7712)\end{array}$} & \multicolumn{2}{|c|}{$\begin{array}{l}2010 \\
(\mathrm{~N}=7159)\end{array}$} & \multicolumn{2}{|c|}{$\begin{array}{l}2014-15 \\
(\mathrm{~N}=6248)\end{array}$} & \multicolumn{2}{|c|}{$\begin{array}{l}2019-20 \\
(\mathrm{~N}=6319)\end{array}$} \\
\hline & $n$ & $\%$ & $n$ & $\%$ & $n$ & $\%$ & $n$ & $\%$ & $n$ & $\%$ \\
\hline East & 1,533 & 22.9 & 1,973 & 25.6 & 1,790 & 25.0 & 1,545 & 24.7 & 1,512 & 23.9 \\
\hline South & 1,490 & 22.2 & 2,014 & 26.1 & 1,771 & 24.7 & 1,503 & 24.1 & 1,432 & 22.7 \\
\hline North & 1,241 & 18.5 & 1,315 & 17.1 & 1,043 & 14.6 & 847 & 13.6 & 963 & 23.9 \\
\hline \multicolumn{11}{|l|}{ Place of delivery } \\
\hline Home/others & 4,723 & 70.5 & 5,429 & 70.5 & 2,005 & 28.0 & 525 & 8.4 & 435 & 6.9 \\
\hline $\begin{array}{l}\text { Public health } \\
\text { facility }\end{array}$ & 1,856 & 27.7 & 2,169 & 28.2 & 5,101 & 71.3 & 5,702 & 91.3 & 5,775 & 91.4 \\
\hline $\begin{array}{l}\text { Private health } \\
\text { facility }\end{array}$ & 121 & 1.8 & 98 & 1.3 & 50 & 0.7 & 18 & 0.3 & 109 & 1.7 \\
\hline
\end{tabular}

\section{Trends in prevalence of CS in Rwanda}

Overall, the rate of CS increased significantly from $2.2 \%$ (95\% Cl 1.8-2.6) in 2000 to $15.6 \%(95 \% \mathrm{Cl} 13.9-16.5)$ in 2019-20. The rate was consistently high among women 15-19 years of age, women residing in urban areas, those with secondary or higher levels of education, from the richest households, with access to information, on paid employment, and those who had one child, male babies, and multiple pregnancies across the years (Table 2).

We found regional disparities in the prevalence of CS across the years. Kigali city had a significantly higher prevalence than the other regions; with a three-fold increase in CS from $8.8 \%$ in 2000 to $26.4 \%$ in $2019-20$. The Northern region had the lowest prevalence of CS despite a 10-fold increase between 2000 (1.3\%) and 2019-20 (13.2\%) (Table 2).

There was an upward trend in the prevalence of CS in both public and private health facilities. The prevalence of CS in public facilities increased by about seven percentage points from $8.2 \%(95 \% \mathrm{Cl} 6.8-10.0)$ in 2000 to $15.4 \%$ (95\% Cl 14.1-16.8) in 2019-20. On the other hand, there was about a four-fold increase in the prevalence of CS in private facilities from $13.6 \%(95 \% \mathrm{Cl} 7.4-23.8 \%)$ in 2000 to $60.6 \%(95 \% \mathrm{Cl} 47.2-72.5)$ in $2019-20$; though the 2019-20 rate was a 20\% decrease from the 2014-15 rate (Table 2). The prevalence of CS between public and private health facilities in 2010, 2014-15 and 2019-20 differed significantly despite having almost similar rates in 2005 (Table 2). 
Table 2

Prevalence of caesarean section according to participants' characteristics

\begin{tabular}{|c|c|c|c|c|c|c|c|c|c|c|}
\hline \multirow[t]{2}{*}{ Characteristics } & \multicolumn{2}{|c|}{2000} & \multicolumn{2}{|c|}{2005} & \multicolumn{2}{|l|}{2010} & \multicolumn{2}{|c|}{ 2014-15 } & \multicolumn{2}{|c|}{$2019-20$} \\
\hline & $\%$ & $\begin{array}{l}95 \% \\
\mathrm{Cl}\end{array}$ & $\%$ & $\begin{array}{l}95 \% \\
\mathrm{Cl}\end{array}$ & $\%$ & $\begin{array}{l}95 \% \\
\mathrm{Cl}\end{array}$ & $\%$ & $\begin{array}{l}95 \% \\
\mathrm{Cl}\end{array}$ & $\%$ & $\begin{array}{l}95 \% \\
\mathrm{Cl}\end{array}$ \\
\hline \multicolumn{11}{|l|}{ Age, years } \\
\hline $15-19$ & 3.7 & $\begin{array}{l}(2.0- \\
6.9)\end{array}$ & 4.1 & $\begin{array}{l}(2.3- \\
7.2)\end{array}$ & 9.9 & $\begin{array}{l}(7.0- \\
13.8)\end{array}$ & 14.1 & $\begin{array}{l}(10.3- \\
18.9)\end{array}$ & 14.2 & $\begin{array}{l}(10.1- \\
19.6)\end{array}$ \\
\hline $20-34$ & 2.2 & $\begin{array}{l}(1.8- \\
2.8)\end{array}$ & 3.1 & $\begin{array}{l}(2.5- \\
3.7)\end{array}$ & 7.4 & $\begin{array}{l}(6.5- \\
8.3)\end{array}$ & 13.3 & $\begin{array}{l}(12.1- \\
14.7)\end{array}$ & 15.8 & $\begin{array}{l}(14.3- \\
17.4)\end{array}$ \\
\hline $35-49$ & 1.5 & $\begin{array}{l}(0.9- \\
2.5)\end{array}$ & 1.8 & $\begin{array}{l}(1.2- \\
2.6)\end{array}$ & 5.2 & $\begin{array}{l}(3.9- \\
6.9)\end{array}$ & 9.7 & $\begin{array}{l}(7.8- \\
12.0)\end{array}$ & 13.5 & $\begin{array}{l}(11.2- \\
16.1)\end{array}$ \\
\hline \multicolumn{11}{|l|}{ Residence } \\
\hline Urban & 6.4 & $\begin{array}{l}(5.0- \\
8.1)\end{array}$ & 7.4 & $\begin{array}{l}(5.5- \\
9.97)\end{array}$ & 16.0 & $\begin{array}{l}(13.0- \\
19.6)\end{array}$ & 22.1 & $\begin{array}{l}(18.9- \\
25.8)\end{array}$ & 14.7 & $\begin{array}{l}(12.4- \\
17.4)\end{array}$ \\
\hline Rural & 1.4 & $\begin{array}{l}(1.1- \\
1.9)\end{array}$ & 2.1 & $\begin{array}{l}(1.7- \\
2.6)\end{array}$ & 6.0 & $\begin{array}{l}(5.3- \\
6.8)\end{array}$ & 10.8 & $\begin{array}{l}(9.7- \\
12.1)\end{array}$ & 15.3 & $\begin{array}{l}(13.9- \\
16.7)\end{array}$ \\
\hline \multicolumn{11}{|l|}{ Marital status } \\
\hline Not in a union & 1.6 & $\begin{array}{l}(1.0- \\
2.7)\end{array}$ & 3.9 & $\begin{array}{l}(2.6- \\
5.7)\end{array}$ & 7.8 & $\begin{array}{l}(6.2- \\
9.7)\end{array}$ & 13.1 & $\begin{array}{l}(10.9- \\
15.7)\end{array}$ & 26.4 & $\begin{array}{l}(22.9- \\
30.3)\end{array}$ \\
\hline Married/cohabiting & 2.2 & $\begin{array}{l}(1.8- \\
2.7)\end{array}$ & 2.7 & $\begin{array}{l}(2.2- \\
3.3)\end{array}$ & 7.0 & $\begin{array}{l}(6.2- \\
7.9)\end{array}$ & 12.7 & $\begin{array}{l}(11.5- \\
14.1)\end{array}$ & 12.9 & $\begin{array}{l}(11.6- \\
14.3)\end{array}$ \\
\hline \multicolumn{11}{|l|}{ Education } \\
\hline No formal & 1.1 & $\begin{array}{l}(0.7- \\
1.7)\end{array}$ & 2.2 & $\begin{array}{l}(1.5- \\
3.1)\end{array}$ & 5.1 & $\begin{array}{l}(3.9- \\
6.8)\end{array}$ & 7.7 & $\begin{array}{l}(5.7- \\
10.3)\end{array}$ & 8.9 & $\begin{array}{l}(6.7- \\
11.9)\end{array}$ \\
\hline Primary & 2.3 & $\begin{array}{l}(1.8- \\
3.0)\end{array}$ & 2.4 & $\begin{array}{l}(1.9- \\
3.1)\end{array}$ & 6.4 & $\begin{array}{l}(5.6- \\
7.2)\end{array}$ & 12.1 & $\begin{array}{l}(10.8- \\
13.5)\end{array}$ & 12.7 & $\begin{array}{l}(11.4- \\
14.2)\end{array}$ \\
\hline Secondary & 5.1 & $\begin{array}{l}(3.7- \\
7.0)\end{array}$ & 8.4 & $\begin{array}{l}(6.1- \\
11.5)\end{array}$ & 18.6 & $\begin{array}{l}(15.0- \\
22.8)\end{array}$ & 22.4 & $\begin{array}{l}(19.1- \\
26.1)\end{array}$ & 24.3 & $\begin{array}{l}(21.1- \\
27.9)\end{array}$ \\
\hline \multicolumn{11}{|l|}{ Occupation } \\
\hline Not working & 7.7 & $\begin{array}{l}(3.2- \\
17.1)\end{array}$ & 4.6 & $\begin{array}{l}(3.4- \\
6.2)\end{array}$ & 9.4 & $\begin{array}{l}(7.4- \\
11.9)\end{array}$ & 13.9 & $\begin{array}{l}(11.2- \\
17.2)\end{array}$ & 19.0 & $\begin{array}{l}(16.4- \\
22.0)\end{array}$ \\
\hline Agriculture & 1.4 & $\begin{array}{l}(1.0- \\
1.8)\end{array}$ & 1.9 & $\begin{array}{l}(1.5- \\
2.4)\end{array}$ & 5.7 & $\begin{array}{l}(5.0- \\
6.6)\end{array}$ & 10.0 & $\begin{array}{l}(8.9- \\
11.2)\end{array}$ & 12.2 & $\begin{array}{l}(10.5- \\
14.0)\end{array}$ \\
\hline $\begin{array}{l}\text { Formal } \\
\text { employment }\end{array}$ & 6.1 & $\begin{array}{l}(4.1- \\
8.9)\end{array}$ & 7.2 & $\begin{array}{l}(5.1- \\
10.1)\end{array}$ & 14.2 & $\begin{array}{l}(11.4- \\
17.6)\end{array}$ & 24.5 & $\begin{array}{l}(20.9- \\
28.5)\end{array}$ & 16.2 & $\begin{array}{l}(14.3- \\
18.3)\end{array}$ \\
\hline
\end{tabular}




\begin{tabular}{|c|c|c|c|c|c|c|c|c|c|c|}
\hline \multirow[t]{2}{*}{ Characteristics } & \multicolumn{2}{|c|}{2000} & \multicolumn{2}{|c|}{2005} & \multicolumn{2}{|c|}{2010} & \multicolumn{2}{|c|}{ 2014-15 } & \multicolumn{2}{|c|}{$2019-20$} \\
\hline & $\%$ & $\begin{array}{l}95 \% \\
\mathrm{Cl}\end{array}$ & $\%$ & $\begin{array}{l}95 \% \\
\mathrm{Cl}\end{array}$ & $\%$ & $\begin{array}{l}95 \% \\
\mathrm{Cl}\end{array}$ & $\%$ & $\begin{array}{l}95 \% \\
\mathrm{Cl}\end{array}$ & $\%$ & $\begin{array}{l}95 \% \\
\mathrm{Cl}\end{array}$ \\
\hline Poorest & 1.6 & $\begin{array}{l}(0.9- \\
2.8)\end{array}$ & 1.0 & $\begin{array}{l}(13.7- \\
18.0)\end{array}$ & 5.5 & $\begin{array}{l}(4.5- \\
6.8)\end{array}$ & 9.7 & $\begin{array}{l}(7.9- \\
11.8)\end{array}$ & 10.4 & $\begin{array}{l}(8.6- \\
12.6)\end{array}$ \\
\hline Poorer & 1.0 & $\begin{array}{l}(0.6- \\
1.8)\end{array}$ & 2.1 & $\begin{array}{l}(1.3- \\
3.3)\end{array}$ & 5.6 & $\begin{array}{l}(4.3- \\
7.2)\end{array}$ & 9.2 & $\begin{array}{l}(7.4- \\
11.4)\end{array}$ & 9.84 & $\begin{array}{l}(8.1- \\
11.9)\end{array}$ \\
\hline Average & 1.6 & $\begin{array}{l}(1.0- \\
2.7)\end{array}$ & 1.8 & $\begin{array}{l}(1.1- \\
2.8)\end{array}$ & 6.5 & $\begin{array}{l}(5.0- \\
8.3)\end{array}$ & 9.9 & $\begin{array}{l}(7.8- \\
12.5)\end{array}$ & 12.5 & $\begin{array}{l}(10.3- \\
15.1)\end{array}$ \\
\hline Richer & 2.2 & $\begin{array}{l}(1.4- \\
3.4)\end{array}$ & 2.5 & $\begin{array}{l}(1.8- \\
3.6)\end{array}$ & 5.8 & $\begin{array}{l}(4.4- \\
7.5)\end{array}$ & 14.4 & $\begin{array}{l}(11.8- \\
17.3)\end{array}$ & 16.8 & $\begin{array}{l}(14.0- \\
20.0)\end{array}$ \\
\hline Richest & 4.3 & $\begin{array}{l}(3.2- \\
5.7)\end{array}$ & 7.8 & $\begin{array}{l}(6.0- \\
10.1)\end{array}$ & 14.2 & $\begin{array}{l}(11.9- \\
16.8)\end{array}$ & 23.2 & $\begin{array}{l}(20.4- \\
26.2)\end{array}$ & 28.3 & $\begin{array}{l}(24.5- \\
32.5)\end{array}$ \\
\hline \multicolumn{11}{|l|}{$\begin{array}{l}\text { ANC attendance, } \\
\text { visits }\end{array}$} \\
\hline $1-3$ & 1.9 & $\begin{array}{l}(1.5- \\
2.4)\end{array}$ & 2.5 & $\begin{array}{l}(2.0- \\
3.0)\end{array}$ & 7.2 & $\begin{array}{l}(6.3- \\
8.2)\end{array}$ & 11.0 & $\begin{array}{l}(9.8- \\
12.3)\end{array}$ & 13.6 & $\begin{array}{l}(12.1- \\
15.3)\end{array}$ \\
\hline$\geq 4$ & 4.9 & $\begin{array}{l}(3.3- \\
7.4)\end{array}$ & 6.8 & $\begin{array}{l}(5.2- \\
9.0)\end{array}$ & 10.4 & $\begin{array}{l}(8.9- \\
12.1)\end{array}$ & 15.5 & $\begin{array}{l}(13.8- \\
17.4)\end{array}$ & 19.6 & $\begin{array}{l}(17.8- \\
21.5)\end{array}$ \\
\hline Missing & 2.1 & $\begin{array}{l}(1.6- \\
2.8)\end{array}$ & 2.6 & $\begin{array}{l}(2.0- \\
3.4)\end{array}$ & 4.9 & $\begin{array}{l}(4.0- \\
6.0)\end{array}$ & 12.2 & $\begin{array}{l}(10.5- \\
14.2)\end{array}$ & 12.2 & $\begin{array}{l}(10.5- \\
14.1)\end{array}$ \\
\hline \multicolumn{11}{|l|}{ Parity } \\
\hline 1 & 4.2 & $\begin{array}{l}(3.0- \\
6.1)\end{array}$ & 6.0 & $\begin{array}{l}(4.6- \\
7.9)\end{array}$ & 15.1 & $\begin{array}{l}(13.1- \\
17.4)\end{array}$ & 17.9 & $\begin{array}{l}(15.7- \\
20.4)\end{array}$ & 20.7 & $\begin{array}{l}(18.4- \\
23.1)\end{array}$ \\
\hline $2-4$ & 2.6 & $\begin{array}{l}(2.0- \\
3.3)\end{array}$ & 3.2 & $\begin{array}{l}(2.5- \\
4.1)\end{array}$ & 7.2 & $\begin{array}{l}(6.1- \\
8.5)\end{array}$ & 13.8 & $\begin{array}{l}(12.2- \\
15.5)\end{array}$ & 16.6 & $\begin{array}{l}(14.9- \\
18.5)\end{array}$ \\
\hline$\geq 5$ & 1.1 & $\begin{array}{l}(0.7- \\
1.6)\end{array}$ & 1.6 & $\begin{array}{l}(1.2- \\
2.3)^{-}\end{array}$ & 2.8 & $\begin{array}{l}(2.2- \\
3.7)\end{array}$ & 6.0 & $\begin{array}{l}(4.6- \\
7.7)\end{array}$ & 7.9 & $\begin{array}{l}(6.4- \\
9.9)\end{array}$ \\
\hline \multicolumn{11}{|l|}{ Baby's sex } \\
\hline Male & 2.4 & $\begin{array}{l}(1.9- \\
3.0)\end{array}$ & 3.2 & $\begin{array}{l}(2.6- \\
3.9)\end{array}$ & 7.9 & $\begin{array}{l}(7.0- \\
9.0)\end{array}$ & 14.2 & $\begin{array}{l}(12.7- \\
15.7)\end{array}$ & 16.2 & $\begin{array}{l}(14.7- \\
17.8)\end{array}$ \\
\hline Female & 1.9 & $\begin{array}{l}(1.4- \\
2.6)\end{array}$ & 2.5 & $\begin{array}{l}(2.0- \\
3.2)\end{array}$ & 6.3 & $\begin{array}{l}(5.5- \\
7.2)\end{array}$ & 11.4 & $\begin{array}{l}(10.0- \\
12.9)\end{array}$ & 14.1 & $\begin{array}{l}(12.4- \\
16.0)\end{array}$ \\
\hline \multicolumn{11}{|c|}{ Baby's birth weight } \\
\hline Average & 6.7 & $\begin{array}{l}(5.3- \\
8.4)\end{array}$ & 7.7 & $\begin{array}{l}(6.3- \\
9.4)\end{array}$ & 9.0 & $\begin{array}{l}(7.9- \\
10.2)\end{array}$ & 12.5 & $\begin{array}{l}\text { (11.3- } \\
13.8)\end{array}$ & 15.4 & $\begin{array}{l}(14.0- \\
16.9)\end{array}$ \\
\hline Low birth weight & 8.8 & $\begin{array}{l}(5.0- \\
15.0)\end{array}$ & 9.5 & $\begin{array}{l}(5.9- \\
15.0)\end{array}$ & 10.0 & $\begin{array}{l}(7.7- \\
12.8)\end{array}$ & 15.2 & $\begin{array}{l}(12.0- \\
19.0)\end{array}$ & 14.7 & $\begin{array}{l}(12.0- \\
17.8)\end{array}$ \\
\hline
\end{tabular}




\begin{tabular}{|c|c|c|c|c|c|c|c|c|c|c|}
\hline \multirow[t]{2}{*}{ Characteristics } & \multicolumn{2}{|c|}{2000} & \multicolumn{2}{|c|}{2005} & \multicolumn{2}{|c|}{2010} & \multicolumn{2}{|c|}{ 2014-15 } & \multicolumn{2}{|c|}{$2019-20$} \\
\hline & $\%$ & $\begin{array}{l}95 \% \\
\mathrm{Cl}\end{array}$ & $\%$ & $\begin{array}{l}95 \% \\
\mathrm{Cl}\end{array}$ & $\%$ & $\begin{array}{l}95 \% \\
\mathrm{Cl}\end{array}$ & $\%$ & $\begin{array}{l}95 \% \\
\mathrm{Cl}\end{array}$ & $\%$ & $\begin{array}{l}95 \% \\
\mathrm{Cl}\end{array}$ \\
\hline Big baby & 0.7 & $\begin{array}{l}(0.5- \\
1.0)\end{array}$ & 1.1 & $\begin{array}{l}(0.8- \\
1.5)\end{array}$ & 4.1 & $\begin{array}{l}(3.3- \\
5.1)\end{array}$ & 12.5 & $\begin{array}{l}(10.3- \\
15.3)\end{array}$ & 14.3 & $\begin{array}{l}(11.4- \\
17.7)\end{array}$ \\
\hline \multicolumn{11}{|l|}{ Twin status } \\
\hline Singleton & 2.0 & $\begin{array}{l}(1.6- \\
2.4)\end{array}$ & 2.8 & $\begin{array}{l}(2.4- \\
3.4)\end{array}$ & 6.8 & $\begin{array}{l}(6.1- \\
7.5)\end{array}$ & 12.0 & $\begin{array}{l}(11.0- \\
13.2)\end{array}$ & 14.8 & $\begin{array}{l}(13.6- \\
16.2)\end{array}$ \\
\hline Multiple & 8.6 & $\begin{array}{l}(3.8- \\
18.4)\end{array}$ & 4.4 & $\begin{array}{l}(1.9- \\
10.1)\end{array}$ & 19.1 & $\begin{array}{l}(12.3- \\
28.4)\end{array}$ & 37.2 & $\begin{array}{l}(27.3- \\
48.4)\end{array}$ & 27.2 & $\begin{array}{l}(18.4- \\
38.3)\end{array}$ \\
\hline \multicolumn{11}{|l|}{$\begin{array}{l}\text { Access to } \\
\text { information }\end{array}$} \\
\hline No & 0.9 & $\begin{array}{l}(0.6- \\
1.4)\end{array}$ & 1.6 & $\begin{array}{l}(1.0- \\
2.6)\end{array}$ & 6.4 & $\begin{array}{l}(4.6- \\
8.9)\end{array}$ & 9.9 & $\begin{array}{l}(7.8- \\
12.5)\end{array}$ & 11.7 & $\begin{array}{l}(9.6- \\
14.1)\end{array}$ \\
\hline Yes & 2.9 & $\begin{array}{l}(2.3- \\
3.6)\end{array}$ & 3.2 & $\begin{array}{l}(2.7- \\
3.9)\end{array}$ & 7.2 & $\begin{array}{l}(6.4- \\
8.0)\end{array}$ & 13.4 & $\begin{array}{l}(12.1- \\
14.7)\end{array}$ & 16.1 & $\begin{array}{l}(14.6- \\
17.7)\end{array}$ \\
\hline \multicolumn{11}{|c|}{ Partner's education } \\
\hline No formal & 1.5 & $\begin{array}{l}(1.0- \\
2.3)\end{array}$ & 1.3 & $\begin{array}{l}(0.8- \\
1.9)\end{array}$ & 5.7 & $\begin{array}{l}(4.3- \\
7.4)\end{array}$ & 9.0 & $\begin{array}{l}(6.8- \\
11.9)\end{array}$ & 8.9 & $\begin{array}{l}(6.5- \\
12.1)\end{array}$ \\
\hline Primary & 1.9 & $\begin{array}{l}(1.5- \\
2.6)\end{array}$ & 2.9 & $\begin{array}{l}(2.2- \\
3.8)\end{array}$ & 6.0 & $\begin{array}{l}(5.2- \\
7.0)\end{array}$ & 11.8 & $\begin{array}{l}(10.5- \\
13.1)\end{array}$ & 13.5 & $\begin{array}{l}(12.0- \\
15.1)\end{array}$ \\
\hline $\begin{array}{l}\text { Secondary and } \\
\text { higher }\end{array}$ & 4.4 & $\begin{array}{l}(2.7- \\
6.9)\end{array}$ & 5.4 & $\begin{array}{l}(4.0- \\
7.1)\end{array}$ & 14.3 & $\begin{array}{l}(11.3- \\
18.0)\end{array}$ & 23.6 & $\begin{array}{l}(20.1- \\
27.7)\end{array}$ & 25.3 & $\begin{array}{l}(21.6- \\
29.3)\end{array}$ \\
\hline \multicolumn{11}{|l|}{ Region } \\
\hline Kigali City & 8.8 & $\begin{array}{l}(6.8- \\
11.5)\end{array}$ & 12.1 & $\begin{array}{l}(8.2- \\
17.5)\end{array}$ & 15.2 & $\begin{array}{l}(11.9- \\
19.1)\end{array}$ & 21.8 & $\begin{array}{l}(18.0- \\
26.0)\end{array}$ & 26.4 & $\begin{array}{l}(22.3- \\
31.0)\end{array}$ \\
\hline South & 2.0 & $\begin{array}{l}(1.3- \\
3.1)\end{array}$ & 2.8 & $\begin{array}{l}(2.1- \\
3.8)\end{array}$ & 7.5 & $\begin{array}{l}(6.0- \\
9.3)\end{array}$ & 14.5 & $\begin{array}{l}(12.1- \\
17.2)\end{array}$ & 16.2 & $\begin{array}{l}(13.8- \\
19.0)\end{array}$ \\
\hline West & 1.9 & $\begin{array}{l}(1.3- \\
2.8)\end{array}$ & 2.8 & $\begin{array}{l}(2.0- \\
3.9)\end{array}$ & 6.1 & $\begin{array}{l}(5.0- \\
7.5)\end{array}$ & 11.4 & $\begin{array}{l}(9.2- \\
14.1)\end{array}$ & 12.5 & $\begin{array}{l}(10.4- \\
14.9)\end{array}$ \\
\hline East & 2.3 & $\begin{array}{l}(1.4- \\
3.5)\end{array}$ & 1.9 & $\begin{array}{l}(1.2- \\
2.9)\end{array}$ & 6.5 & $\begin{array}{l}(5.2- \\
8.0)\end{array}$ & 10.8 & $\begin{array}{l}(9.0- \\
13.0)\end{array}$ & 11.2 & $\begin{array}{l}(8.67- \\
14.4)\end{array}$ \\
\hline North & 1.3 & $\begin{array}{l}(0.8- \\
2.3)\end{array}$ & 1.2 & $\begin{array}{l}(0.7- \\
2.1)\end{array}$ & 4.5 & $\begin{array}{l}(3.1- \\
6.5)\end{array}$ & 8.5 & $\begin{array}{l}(6.4- \\
11.1)\end{array}$ & 13.2 & $\begin{array}{l}(10.7- \\
16.3)\end{array}$ \\
\hline \multicolumn{11}{|l|}{ Type of facility } \\
\hline Public & 8.2 & $\begin{array}{l}(6.8- \\
10.0)\end{array}$ & 10.2 & $\begin{array}{l}(8.6- \\
11.9)\end{array}$ & 9.6 & $\begin{array}{l}(8.7- \\
10.7)\end{array}$ & 13.9 & $\begin{array}{l}(12.6- \\
15.2)\end{array}$ & 15.4 & $\begin{array}{l}(14.1- \\
16.8)\end{array}$ \\
\hline
\end{tabular}




\begin{tabular}{|c|c|c|c|c|c|c|c|c|c|c|}
\hline \multirow[t]{2}{*}{ Characteristics } & \multicolumn{2}{|c|}{2000} & \multicolumn{2}{|c|}{2005} & \multicolumn{2}{|l|}{2010} & \multicolumn{2}{|c|}{ 2014-15 } & \multicolumn{2}{|c|}{$2019-20$} \\
\hline & $\%$ & $\begin{array}{l}95 \% \\
\mathrm{Cl}\end{array}$ & $\%$ & $\begin{array}{l}95 \% \\
\mathrm{Cl}\end{array}$ & $\%$ & $\begin{array}{l}95 \% \\
\mathrm{Cl}\end{array}$ & $\%$ & $\begin{array}{l}95 \% \\
\mathrm{Cl}\end{array}$ & $\%$ & $\begin{array}{l}95 \% \\
\mathrm{Cl}\end{array}$ \\
\hline Private & 13.6 & $\begin{array}{l}(7.4- \\
23.8)\end{array}$ & 10.9 & $\begin{array}{l}(5.7- \\
20.0)\end{array}$ & 41.5 & $\begin{array}{l}(26.6- \\
58.1)\end{array}$ & 76.5 & $\begin{array}{l}(36.9- \\
94.8)\end{array}$ & 60.6 & $\begin{array}{l}(47.2- \\
72.5)\end{array}$ \\
\hline
\end{tabular}

\section{Factors associated with CS in Rwanda}

Based on the unadjusted logistic regression models, there was an association between CS and residential area, education, employment, wealth, women's age, access to media, parity, ANC attendance, multiple births, baby's sex and birth weight (Suppl. Table 2).

Table 3 presents the results of the population-based multivariable logistic regression models for each of the four surveys and the pooled model. Results from the pooled model indicate an association between CS and maternal age, occupation, wealth, ANC attendance, parity, sex and size of the child, and region of residence. The odds of CS were higher among women aged 35 years or older (aOR: 1.77, 95\% Cl 1.26-2.49) and those who had attended four or more ANC visits (aOR: $1.64,95 \% \mathrm{Cl} 1.46-1.84$ ) compared to women 15-19 years of age and those who had visited ANC less than four times, respectively. Moreover, women in formal employment (aOR: $1.21,95 \% \mathrm{Cl} 1.00-1.46$ ), those with primary (aOR: $1.24,95 \% \mathrm{Cl} 1.03-1.51$ ) and secondary or higher levels of education (aOR: $1.58,95 \% \mathrm{Cl} 1.24-2.04$ ) and from the richest households (aOR: $1.52,95 \% \mathrm{Cl} 1.23-$ 1.87) had higher odds of CS compared to unemployed, uneducated, and poorest women, respectively. On the other hand, multiparous women and those residing outside Kigali City had $34-75 \%$ and $30-53 \%$ lower odds of $\mathrm{CS}$, respectively. Besides, women who delivered big babies had lower odds of CS (aOR: $0.42,95 \% \mathrm{Cl} 0.36-0.48$ ) compared to those with normal-weight babies (Table 3).

Across the surveys, women with multiple pregnancies, with $\geq 4$ ANC visits and from the richest households had higher odds of CS while multiparous women and women with female babies had lower odds of CS. In 2000, women working in the agricultural sector had lower odds of CS while in 2014, women in formal employment had higher odds of CS. Region of residence and partner education, and marital status were associated with CS in 2005 and 2010, respectively (Table 3). 
Table 3

Factors associated with caesarean section at a population level in Rwanda

\begin{tabular}{|c|c|c|c|c|c|c|}
\hline Characteristics & 2000 & 2005 & 2010 & 2014 & $2019-20$ & Pooled \\
\hline & $\begin{array}{l}\text { aOR }(95 \% \\
\mathrm{Cl})\end{array}$ & $\begin{array}{l}\text { aOR }(95 \% \\
\mathrm{Cl})\end{array}$ & $\begin{array}{l}\text { aOR }(95 \% \\
\mathrm{Cl})\end{array}$ & $\begin{array}{l}\text { aOR }(95 \% \\
\mathrm{Cl})\end{array}$ & $\begin{array}{l}\text { aOR }(95 \% \\
\mathrm{Cl})\end{array}$ & $\begin{array}{l}\text { aOR }(95 \% \\
\mathrm{Cl})\end{array}$ \\
\hline
\end{tabular}

\section{Age, years}

$\begin{array}{lllllll}15-19 & 1 & 1 & 1 & 1 & 1 & 1 \\ 20-34 & 0.76(0.32- & 1.05 & 1.18(0.67- & 1.12(0.72- & 1.17 & 1.12 \\ & 1.78) & (0.42- & 2.05) & 1.72) & 0.60- & (0.83- \\ & & 2.60) & & & 2.25) & 1.50) \\ 35-49 & 1.13(0.31- & 0.92 & 1.86(0.91- & 1.45(0.86- & 1.67 & 1.77 \\ & 4.07) & \begin{array}{l}(0.33- \\ 2.51)\end{array} & 3.79) & 2.45) & (0.82- & (1.26- \\ & & & & & 3.37) & 2.49)^{\star}\end{array}$

\section{Residence}

$\begin{array}{lllllll}\text { Urban } & 1 & 1 & 1 & 1 & 1 & 1 \\ \text { Rural } & 1.03(0.45- & 1.07 & 0.61(0.34- & 0.86(0.59- & 0.80 & 0.89 \\ & 2.35) & (0.59- & 1.09) & 1.24) & (0.58- & (0.72- \\ & & 1.95) & & & 1.12) & 1.09)\end{array}$

\section{Marital status $\uparrow$}

$\begin{array}{llllll}\text { Not in a union } & 1 & 1 & 1 & 1 & 1 \\ \text { Married/cohabiting } & 1.08(0.48- & 0.70 & 1.97(1.23- & 1.01(0.73- & 1.27 \\ & 2.43) & \begin{array}{l}(0.37- \\ 1.35)\end{array} & 3.12)^{\star \star} & 1.40) & (1.00- \\ & & & & & 1.61)\end{array}$

\section{Education}

\begin{tabular}{|c|c|c|c|c|c|c|}
\hline No formal & 1 & 1 & 1 & 1 & 1 & 1 \\
\hline Primary & $\begin{array}{l}1.66(0.82- \\
3.34)\end{array}$ & $\begin{array}{l}0.63 \\
(0.39- \\
1.00)\end{array}$ & $\begin{array}{l}1.05(0.72- \\
1.53)\end{array}$ & $\begin{array}{l}1.27(0.88- \\
1.83)\end{array}$ & $\begin{array}{l}1.14 \\
(0.75- \\
1.73)\end{array}$ & $\begin{array}{l}1.24 \\
(1.03- \\
1.51)^{\star}\end{array}$ \\
\hline Secondary & $\begin{array}{l}2.68(0.62- \\
4.57)\end{array}$ & $\begin{array}{l}1.04 \\
(0.57- \\
1.91)\end{array}$ & $\begin{array}{l}1.83(1.06- \\
3.18)^{\star}\end{array}$ & $\begin{array}{l}1.30(0.83- \\
2.05)\end{array}$ & $\begin{array}{l}1.26 \\
(0.76- \\
2.08)\end{array}$ & $\begin{array}{l}1.58 \\
(1.24- \\
2.04)^{\star}\end{array}$ \\
\hline \multicolumn{7}{|l|}{ Occupation } \\
\hline Not working & 1 & 1 & 1 & 1 & 1 & 1 \\
\hline Agriculture & $\begin{array}{l}0.27(0.10- \\
0.72)^{\star \star}\end{array}$ & $\begin{array}{l}0.76 \\
(0.49- \\
1.17)\end{array}$ & $\begin{array}{l}0.93(0.62- \\
1.38)\end{array}$ & $\begin{array}{l}1.02(0.69- \\
1.52)\end{array}$ & $\begin{array}{l}1.00 \\
(0.74- \\
1.35)\end{array}$ & $\begin{array}{l}0.78 \\
(0.66- \\
0.93)^{\star}\end{array}$ \\
\hline $\begin{array}{l}\text { Formal } \\
\text { employment }\end{array}$ & $\begin{array}{l}0.39(0.11- \\
1.41)\end{array}$ & $\begin{array}{l}0.72 \\
(0.42- \\
1.25)\end{array}$ & $\begin{array}{l}1.34(0.89- \\
2.01)\end{array}$ & $\begin{array}{l}1.92(1.34- \\
2.77)^{\star \star \star}\end{array}$ & $\begin{array}{l}1.00(0 \\
.75-1.32)\end{array}$ & $\begin{array}{l}1.21 \\
(1.00- \\
1.46)^{\star}\end{array}$ \\
\hline
\end{tabular}

${ }^{*} \mathrm{p}<0.05 ; * \star \mathrm{p}<0.01 ; * \star * \mathrm{p}<0.001 ; \mathrm{ANC}$, antenatal care; $\mathrm{Cl}$, confidence interval; $\uparrow$ Marital status was excluded in 2019-20 due to multicollinearity 


\begin{tabular}{|c|c|c|c|c|c|c|}
\hline Characteristics & 2000 & 2005 & 2010 & 2014 & $2019-20$ & Pooled \\
\hline \multicolumn{7}{|l|}{ Wealth, quintiles } \\
\hline Poorest & 1 & 1 & 1 & 1 & 1 & 1 \\
\hline Poorer & $\begin{array}{l}0.88(0.34- \\
2.29)\end{array}$ & $\begin{array}{l}1.77 \\
(0.84- \\
3.72)\end{array}$ & $\begin{array}{l}1.02(0.69- \\
1.52)\end{array}$ & $\begin{array}{l}0.90(0.63- \\
1.30)\end{array}$ & $\begin{array}{l}0.92 \\
(0.64- \\
1.32)\end{array}$ & $\begin{array}{l}0.91 \\
(0.75- \\
1.11)\end{array}$ \\
\hline Average & $\begin{array}{l}1.06(0.38- \\
2.99)\end{array}$ & $\begin{array}{l}1.35 \\
(0.61- \\
3.00)\end{array}$ & $\begin{array}{l}1.23(0.84- \\
1.80)\end{array}$ & $\begin{array}{l}1.02(0.70- \\
1.49)\end{array}$ & $\begin{array}{l}1.18 \\
(0.82- \\
1.18)\end{array}$ & $\begin{array}{l}1.06 \\
(0.86- \\
1.29)\end{array}$ \\
\hline Richer & $\begin{array}{l}1.28(0.46- \\
3.56)\end{array}$ & $\begin{array}{l}1.65 \\
(0.79- \\
3.43)\end{array}$ & $\begin{array}{l}1.02(0.68- \\
1.52)\end{array}$ & $\begin{array}{l}1.51(1.06- \\
2.16)^{\star}\end{array}$ & $\begin{array}{l}1.50 \\
(1.03- \\
2.21)^{\star \star}\end{array}$ & $\begin{array}{l}1.18 \\
(0.97- \\
1.44)\end{array}$ \\
\hline Richest & $\begin{array}{l}1.69(0.68- \\
4.21)\end{array}$ & $\begin{array}{l}2.78 \\
(1.19- \\
6.50)^{\star}\end{array}$ & $\begin{array}{l}1.44(0.87- \\
2.39)\end{array}$ & $\begin{array}{l}1.76(1.15- \\
2.71)^{\star}\end{array}$ & $\begin{array}{l}2.43 \\
(1.58- \\
3.72)^{\star \star}\end{array}$ & $\begin{array}{l}1.52 \\
(1.23- \\
1.87)^{\star}\end{array}$ \\
\hline \multicolumn{7}{|l|}{$\begin{array}{l}\text { ANC attendance, } \\
\text { visits }\end{array}$} \\
\hline $1-3$ & 1 & 1 & 1 & 1 & 1 & 1 \\
\hline$\geq 4$ & $\begin{array}{l}1.19(0.65- \\
2.16)\end{array}$ & $\begin{array}{l}1.86 \\
(1.22- \\
2.83)^{\star \star}\end{array}$ & $\begin{array}{l}1.18(0.93- \\
1.52)\end{array}$ & $\begin{array}{l}1.27(1.04- \\
1.57)^{\star}\end{array}$ & $\begin{array}{l}1.44 \\
(1.18- \\
1.75)^{\star}\end{array}$ & $\begin{array}{l}1.64 \\
(1.46- \\
1.84)^{\star}\end{array}$ \\
\hline Missing & $\begin{array}{l}0.89(0.59- \\
1.34)\end{array}$ & $\begin{array}{l}1.16 \\
(0.88- \\
1.53)\end{array}$ & $\begin{array}{l}0.82(0.67- \\
1.01)\end{array}$ & $\begin{array}{l}1.12(0.93- \\
1.34)\end{array}$ & $\begin{array}{l}0.91 \\
(0.76- \\
1.09)\end{array}$ & $\begin{array}{l}1.00 \\
(0.90- \\
1.10)\end{array}$ \\
\hline \multicolumn{7}{|l|}{ Parity } \\
\hline 1 & 1 & 1 & 1 & 1 & 1 & 1 \\
\hline $2-4$ & $\begin{array}{l}0.45(0.25- \\
0.84)^{\star}\end{array}$ & $\begin{array}{l}0.83 \\
(0.49- \\
1.39)\end{array}$ & $\begin{array}{l}0.48(0.37- \\
0.64)^{\star \star \star}\end{array}$ & $\begin{array}{l}0.64(0.50- \\
0.81)^{\star \star \star}\end{array}$ & $\begin{array}{l}0.84 \\
(0.64- \\
1.11)\end{array}$ & $\begin{array}{l}0.66 \\
(0.57- \\
0.75)^{\star \star \star}\end{array}$ \\
\hline $5+$ & $\begin{array}{l}0.22(0.08- \\
0.61)^{\star \star}\end{array}$ & $\begin{array}{l}0.53 \\
(0.27- \\
1.01)\end{array}$ & $\begin{array}{l}0.15(0.10- \\
0.23)^{\star \star \star}\end{array}$ & $\begin{array}{l}0.22(0.14- \\
0.34)^{\star \star \star}\end{array}$ & $\begin{array}{l}0.35 \\
(0.23- \\
0.54)^{\star \star}\end{array}$ & $\begin{array}{l}0.25 \\
(0.20- \\
0.31)^{\star \star \star}\end{array}$ \\
\hline \multicolumn{7}{|l|}{ Baby's sex } \\
\hline Male & 1 & 1 & 1 & 1 & 1 & 1 \\
\hline Female & $\begin{array}{l}0.80(0.55- \\
1.18)\end{array}$ & $\begin{array}{l}0.73 \\
(0.55- \\
0.97)^{\star}\end{array}$ & $\begin{array}{l}0.81(0.67- \\
0.97)^{\star}\end{array}$ & $\begin{array}{l}0.79(0.67- \\
0.94)^{\star \star}\end{array}$ & $\begin{array}{l}0.81 \\
(0.68- \\
0.96)^{\star}\end{array}$ & $\begin{array}{l}0.78 \\
(0.71- \\
0.86)^{\star}\end{array}$ \\
\hline \multicolumn{7}{|c|}{ Baby's birth weight } \\
\hline Average & 1 & 1 & 1 & 1 & 1 & 1 \\
\hline
\end{tabular}




\begin{tabular}{|c|c|c|c|c|c|c|}
\hline Characteristics & 2000 & 2005 & 2010 & 2014 & 2019-20 & Pooled \\
\hline Low birth weight & $\begin{array}{l}1.49(0.68- \\
3.27)\end{array}$ & $\begin{array}{l}1.31 \\
(0.70- \\
2.45)\end{array}$ & $\begin{array}{l}0.78(0.53- \\
1.14)\end{array}$ & $\begin{array}{l}1.06(0.77- \\
1.44)\end{array}$ & $\begin{array}{l}0.99 \\
(0.73- \\
1.34)\end{array}$ & $\begin{array}{l}1.03 \\
(0.85- \\
1.24)\end{array}$ \\
\hline Big baby & $\begin{array}{l}0.14(0.07- \\
0.26)^{\star \star \star}\end{array}$ & $\begin{array}{l}0.20 \\
(0.13- \\
0.32)^{\star \star \star}\end{array}$ & $\begin{array}{l}0.59(0.45- \\
0.78)\end{array}$ & $\begin{array}{l}1.23(0.96- \\
1.58)\end{array}$ & $\begin{array}{l}1.31 \\
(0.97- \\
1.77)\end{array}$ & $\begin{array}{l}0.42 \\
(0.36- \\
0.48)^{\star \star \star}\end{array}$ \\
\hline \multicolumn{7}{|l|}{ Twin status } \\
\hline Singleton & 1 & 1 & 1 & 1 & 1 & 1 \\
\hline Multiple & $\begin{array}{l}8.55(3.36- \\
21.76)^{\star \star \star}\end{array}$ & $\begin{array}{l}1.57 \\
(0.57- \\
4.32)\end{array}$ & $\begin{array}{l}6.11(3.27- \\
11.40) \star \star \star\end{array}$ & $\begin{array}{l}6.49(3.93- \\
10.74)^{\star \star \star}\end{array}$ & $\begin{array}{l}3.24 \\
(1.84- \\
5.70)^{\star \star}\end{array}$ & $\begin{array}{l}4.53 \\
(3.38- \\
6.06)^{\star \star}\end{array}$ \\
\hline \multicolumn{7}{|l|}{$\begin{array}{l}\text { Access to } \\
\text { information }\end{array}$} \\
\hline No & 1 & 1 & 1 & 1 & 1 & 1 \\
\hline Yes & $\begin{array}{l}1.45(0.70- \\
2.99)\end{array}$ & $\begin{array}{l}1.27 \\
(0.71- \\
2.28)\end{array}$ & $\begin{array}{l}0.75(0.48- \\
1.17)\end{array}$ & $\begin{array}{l}0.98(0.70- \\
1.38)\end{array}$ & $\begin{array}{l}0.92 \\
(0.68- \\
1.24)\end{array}$ & $\begin{array}{l}1.06 \\
(0.88- \\
1.27)\end{array}$ \\
\hline \multicolumn{7}{|c|}{ Partner's education } \\
\hline No formal & 1 & 1 & 1 & 1 & 1 & 1 \\
\hline Primary & $\begin{array}{l}0.60(0.32- \\
1.13)\end{array}$ & $\begin{array}{l}1.79 \\
(1.07- \\
2.99)^{\star}\end{array}$ & $\begin{array}{l}0.78(0.55- \\
1.10)\end{array}$ & $\begin{array}{l}1.01(0.74- \\
1.36)^{(1)}\end{array}$ & $\begin{array}{l}1.23 \\
(0.85- \\
1.80)\end{array}$ & $\begin{array}{l}1.10 \\
(0.92- \\
1.31)\end{array}$ \\
\hline $\begin{array}{l}\text { Secondary and } \\
\text { higher }\end{array}$ & $\begin{array}{l}0.47(0.21- \\
1.06)\end{array}$ & $\begin{array}{l}1.03 \\
(0.55- \\
1.95)\end{array}$ & $\begin{array}{l}0.90(0.53- \\
1.54)\end{array}$ & $\begin{array}{l}1.15(0.77- \\
1.71)\end{array}$ & $\begin{array}{l}1.40 \\
(0.87- \\
2.25)\end{array}$ & $\begin{array}{l}1.08 \\
(0.85- \\
1.36)\end{array}$ \\
\hline \multicolumn{7}{|l|}{ Region } \\
\hline Kigali City & 1 & 1 & 1 & 1 & 1 & 1 \\
\hline South & $\begin{array}{l}0.61(0.25- \\
1.49)\end{array}$ & $\begin{array}{l}0.46 \\
(0.23- \\
0.92)^{\star}\end{array}$ & $\begin{array}{l}1.10(0.51- \\
2.39)\end{array}$ & $\begin{array}{l}1.10(0.70- \\
1.72)\end{array}$ & $\begin{array}{l}1.01 \\
(0.69- \\
1.49)\end{array}$ & $\begin{array}{l}0.76 \\
(0.59- \\
0.97)^{\star}\end{array}$ \\
\hline West & $\begin{array}{l}0.84(0.38- \\
1.89)\end{array}$ & $\begin{array}{l}0.56 \\
(0.26- \\
1.18)\end{array}$ & $\begin{array}{l}0.97(0.43- \\
2.19)\end{array}$ & $\begin{array}{l}0.98(0.65- \\
1.48)\end{array}$ & $\begin{array}{l}0.73 \\
(0.49- \\
1.07)\end{array}$ & $\begin{array}{l}0.70 \\
(0.54- \\
0.90)^{\star}\end{array}$ \\
\hline
\end{tabular}

${ }^{*} \mathrm{p}<0.05 ; * \star \mathrm{p}<0.01 ; * * \mathrm{k}<0.001 ; \mathrm{ANC}$, antenatal care; $\mathrm{Cl}$, confidence interval; $\uparrow$ Marital status was excluded in 2019-20 due to multicollinearity 


\begin{tabular}{|c|c|c|c|c|c|c|}
\hline Characteristics & 2000 & 2005 & 2010 & 2014 & $2019-20$ & Pooled \\
\hline East & $\begin{array}{l}0.74(0.31- \\
1.73)\end{array}$ & $\begin{array}{l}0.36 \\
(0.16- \\
0.80)^{*}\end{array}$ & $\begin{array}{l}1.04(0.46- \\
2.32)\end{array}$ & $\begin{array}{l}0.90(0.59- \\
1.38)\end{array}$ & $\begin{array}{l}0.74 \\
(0.50- \\
1.10)\end{array}$ & $\begin{array}{l}0.71 \\
(0.55- \\
0.91)^{\star}\end{array}$ \\
\hline North & $\begin{array}{l}0.45(0.17- \\
1.19)\end{array}$ & $\begin{array}{l}0.22 \\
(0.10- \\
0.51)^{\star \star \star}\end{array}$ & $\begin{array}{l}0.74(0.31- \\
1.75)\end{array}$ & $\begin{array}{l}0.66(0.42- \\
1.05)\end{array}$ & $\begin{array}{l}0.62 \\
(0.40- \\
0.96)^{\star}\end{array}$ & $\begin{array}{l}0.47 \\
(0.35- \\
0.62)^{\star \star \star}\end{array}$ \\
\hline
\end{tabular}

The results from the hospital-based model revealed a similar direction of association as those of the population-based model except for higher odds of CS among women who delivered big babies in 2000 (aOR: 2.00, 95\% Cl 1.11-3.61), 2014-15 (aOR: 1.57, 95\% Cl 1.22-2.03), 2019-20 (aOR: 1.84, 95\% Cl 1.34-1.52) and the pooled model (aOR: $1.45,95 \% \mathrm{Cl} 1.26-1.66)$ (Table 4). 
Table 4

Factors associated with caesarean section at the health facility level in Rwanda

\begin{tabular}{|c|c|c|c|c|c|c|}
\hline Characteristics & 2000 & 2005 & 2010 & 2014 & $2019-20$ & Pooled \\
\hline & $\begin{array}{l}\mathrm{aOR}(95 \% \\
\mathrm{Cl})\end{array}$ & $\begin{array}{l}\mathrm{aOR}(95 \% \\
\mathrm{Cl})\end{array}$ & $\begin{array}{l}\mathrm{aOR}(95 \% \\
\mathrm{Cl})\end{array}$ & $\begin{array}{l}\text { aOR }(95 \% \\
\mathrm{Cl})\end{array}$ & $\begin{array}{l}\mathrm{aOR}(95 \% \\
\mathrm{Cl})\end{array}$ & $\begin{array}{l}\text { aOR }(95 \% \\
\mathrm{Cl})\end{array}$ \\
\hline
\end{tabular}

\section{Age, years}

$\begin{array}{lllllll}15-19 & 1 & 1 & 1 & 1 & 1 & 1 \\ 20-34 & 0.69(0.29- & 1.26 & 1.23(0.70- & 1.09(0.71- & 1.18 & 1.13 \\ & 1.64) & (0.54- & 2.15) & 1.70) & (0.61- & (0.84- \\ & & 2.92) & & & 2.25) & 1.51) \\ 35-49 & 1.06(0.26- & 1.08 & 1.97(0.95- & 1.43(0.84- & 1.72 & 1.78 \\ & 4.22) & (0.41- & 4.09) & 2.42) & (0.86- & (1.26- \\ & & 2.83) & & & 3.45) & 2.51)^{*}\end{array}$

\section{Marital status $†$}

$\begin{array}{llllll}\text { Not in a union } & 1 & 1 & 1 & 1 & 1 \\ \text { Married/cohabiting } & 1.18(0.51- & 0.63 & 1.87(1.16- & 1.03(0.74- & 1.21 \\ & 2.76) & (0.33- & 3.00)^{*} & 1.42) & (0.95- \\ & & 1.23) & & & 1.54)\end{array}$

\section{Access to} information

$\begin{array}{lllllll}\text { No } & 1 & 1 & 1 & 1 & 1 & 1 \\ \text { Yes } & 1.31(0.62- & 1.29 & 0.73(0.47- & 0.95(0.68- & 0.93 & 0.94 \\ & 2.79) & (0.69- & 1.14) & 1.34) & (0.69- & (0.78- \\ & & 2.41) & & & 1.26) & 1.13)\end{array}$

\section{Education}

\begin{tabular}{|c|c|c|c|c|c|c|}
\hline No formal & 1 & 1 & 1 & 1 & 1 & 1 \\
\hline Primary & $\begin{array}{l}1.68(0.78- \\
3.63)\end{array}$ & $\begin{array}{l}0.56 \\
(0.34- \\
0.90)^{\star}\end{array}$ & $\begin{array}{l}1.00(0.69- \\
1.46)\end{array}$ & $\begin{array}{l}1.21(0.84- \\
1.75)\end{array}$ & $\begin{array}{l}1.06 \\
(0.69- \\
1.63)\end{array}$ & $\begin{array}{l}1.08 \\
(0.89- \\
1.31)\end{array}$ \\
\hline Secondary & $\begin{array}{l}1.68(0.56- \\
5.06)\end{array}$ & $\begin{array}{l}0.89 \\
(0.49- \\
1.61)\end{array}$ & $\begin{array}{l}1.69(0.98- \\
2.92)\end{array}$ & $\begin{array}{l}1.26(0.81- \\
1.96)\end{array}$ & $\begin{array}{l}1.18 \\
(0.71- \\
1.97)\end{array}$ & $\begin{array}{l}1.37 \\
(1.06- \\
1.75)^{*}\end{array}$ \\
\hline
\end{tabular}

\section{Occupation}

\begin{tabular}{|c|c|c|c|c|c|c|}
\hline Not working & 1 & 1 & 1 & 1 & 1 & 1 \\
\hline Agriculture & $\begin{array}{l}0.31(0.11- \\
0.92)^{\star}\end{array}$ & $\begin{array}{l}0.83 \\
(0.53- \\
1.30)\end{array}$ & $\begin{array}{l}0.91(0.61- \\
1.36)\end{array}$ & $\begin{array}{l}1.04(0.70- \\
1.55)\end{array}$ & $\begin{array}{l}1.02 \\
(0.76- \\
1.37)\end{array}$ & $\begin{array}{l}0.82 \\
(0.69- \\
0.98)\end{array}$ \\
\hline $\begin{array}{l}\text { Formal } \\
\text { employment }\end{array}$ & $\begin{array}{l}0.42(0.12- \\
1.46)\end{array}$ & $\begin{array}{l}0.76 \\
(0.45- \\
1.29)\end{array}$ & $\begin{array}{l}1.24(0.82- \\
1.87)\end{array}$ & $\begin{array}{l}1.93(1.34- \\
2.79)^{\star \star \star}\end{array}$ & $\begin{array}{l}1.01 \\
(0.76- \\
1.33)\end{array}$ & $\begin{array}{l}1.12 \\
(0.93- \\
1.35)^{*}\end{array}$ \\
\hline
\end{tabular}

${ }^{*} \mathrm{p}<0.05 ;{ }^{* *} \mathrm{p}<0.01 ;{ }^{* * *} \mathrm{p}<0.001 ;+$ Marital status was excluded in 2019-20 due to multicollinearity 


\begin{tabular}{|c|c|c|c|c|c|c|}
\hline Characteristics & 2000 & 2005 & 2010 & 2014 & $2019-20$ & Pooled \\
\hline \multicolumn{7}{|l|}{ Wealth, quintiles } \\
\hline Poorest & 1 & 1 & 1 & 1 & 1 & 1 \\
\hline Poorer & $\begin{array}{l}0.92(0.32- \\
2.60)\end{array}$ & $\begin{array}{l}1.57 \\
(0.71- \\
3.45)\end{array}$ & $\begin{array}{l}1.05(0.70- \\
1.57)\end{array}$ & $\begin{array}{l}0.84(0.58- \\
1.20)\end{array}$ & $\begin{array}{l}0.86 \\
(0.60- \\
1.23)\end{array}$ & $\begin{array}{l}0.94 \\
(0.77- \\
1.15)\end{array}$ \\
\hline Average & $\begin{array}{l}0.89(0.31- \\
2.57)\end{array}$ & $\begin{array}{l}1.22 \\
(0.53- \\
2.80)\end{array}$ & $\begin{array}{l}1.21(0.82- \\
1.78)\end{array}$ & $\begin{array}{l}0.95(0.65- \\
1.39)\end{array}$ & $\begin{array}{l}1.07 \\
(0.74- \\
1.53)\end{array}$ & $\begin{array}{l}1.04 \\
(0.84- \\
1.27)\end{array}$ \\
\hline Richer & $\begin{array}{l}1.32(0.47- \\
3.73)\end{array}$ & $\begin{array}{l}1.27 \\
(0.62- \\
2.62)\end{array}$ & $\begin{array}{l}0.98(0.65- \\
1.48)\end{array}$ & $\begin{array}{l}1.37(0.96- \\
1.97)\end{array}$ & $\begin{array}{l}1.31 \\
(0.89- \\
1.91)\end{array}$ & $\begin{array}{l}1.20 \\
(0.98- \\
1.46)\end{array}$ \\
\hline Richest & $\begin{array}{l}1.56(0.64- \\
3.81)\end{array}$ & $\begin{array}{l}1.82 \\
(0.77- \\
4.29)\end{array}$ & $\begin{array}{l}1.27(0.77- \\
2.10)\end{array}$ & $\begin{array}{l}1.57(1.02- \\
2.43)^{\star}\end{array}$ & $\begin{array}{l}2.10 \\
(1.38- \\
3.19)^{\star \star}\end{array}$ & $\begin{array}{l}1.53 \\
(1.23- \\
1.89)^{\star \star}\end{array}$ \\
\hline \multicolumn{7}{|l|}{$\begin{array}{l}\text { ANC attendance, } \\
\text { visits }\end{array}$} \\
\hline $1-3$ & 1 & 1 & 1 & 1 & 1 & 1 \\
\hline$\geq 4$ & $\begin{array}{l}1.11(0.61- \\
2.03)\end{array}$ & $\begin{array}{l}1.56 \\
(1.02- \\
2.39)^{\star}\end{array}$ & $\begin{array}{l}1.09(0.85- \\
1.39)^{(0.85}\end{array}$ & $\begin{array}{l}1.22(0.99- \\
1.50)\end{array}$ & $\begin{array}{l}1.38 \\
(1.13- \\
1.68)^{\star}\end{array}$ & $\begin{array}{l}1.39 \\
(1.24- \\
1.56)^{\star \star \star}\end{array}$ \\
\hline Missing & $\begin{array}{l}0.85(0.56- \\
1.30)\end{array}$ & $\begin{array}{l}1.07 \\
(0.80- \\
1.44)\end{array}$ & $\begin{array}{l}0.86(0.70- \\
1.06)\end{array}$ & $\begin{array}{l}1.08(0.90- \\
1.30)\end{array}$ & $\begin{array}{l}0.88 \\
(0.73- \\
1.06)\end{array}$ & $\begin{array}{l}0.95 \\
(0.85- \\
1.05)\end{array}$ \\
\hline \multicolumn{7}{|l|}{ Parity } \\
\hline 1 & 1 & 1 & 1 & 1 & 1 & 1 \\
\hline $2-4$ & $\begin{array}{l}0.53(0.29- \\
0.97)^{\star}\end{array}$ & $\begin{array}{l}1.17 \\
(0.70- \\
1.96)\end{array}$ & $\begin{array}{l}0.53(0.40- \\
0.70)^{\star \star \star}\end{array}$ & $\begin{array}{l}0.65(0.51- \\
0.82)^{\star \star \star}\end{array}$ & $\begin{array}{l}0.86 \\
(0.66- \\
1.12)\end{array}$ & $\begin{array}{l}0.70 \\
(0.61- \\
0.81)^{\star \star \star}\end{array}$ \\
\hline $5+$ & $\begin{array}{l}0.29(0.10- \\
0.86)^{\star}\end{array}$ & $\begin{array}{l}0.87 \\
(0.45- \\
1.68)\end{array}$ & $\begin{array}{l}0.19(0.13- \\
0.29)^{\star \star \star}\end{array}$ & $\begin{array}{l}0.25(0.16- \\
0.38)^{\star \star \star}\end{array}$ & $\begin{array}{l}0.38 \\
(0.25- \\
0.58)^{\star \star}\end{array}$ & $\begin{array}{l}0.30 \\
(0.24- \\
0.37)^{\star \star \star}\end{array}$ \\
\hline \multicolumn{7}{|l|}{ Baby's sex } \\
\hline Male & 1 & 1 & 1 & 1 & 1 & 1 \\
\hline Female & $\begin{array}{l}0.83(0.55- \\
1.24)\end{array}$ & $\begin{array}{l}0.82 \\
(0.61- \\
1.10)\end{array}$ & $\begin{array}{l}0.85(0.70- \\
1.03)\end{array}$ & $\begin{array}{l}0.82(0.69- \\
0.97)^{\star}\end{array}$ & $\begin{array}{l}0.82 \\
(0.69- \\
0.97)^{\star}\end{array}$ & $\begin{array}{l}0.82 \\
(0.75- \\
0.91)^{\star \star}\end{array}$ \\
\hline \multicolumn{7}{|c|}{ Baby's birth weight } \\
\hline Average & 1 & 1 & 1 & 1 & 1 & 1 \\
\hline
\end{tabular}

${ }^{*} \mathrm{p}<0.05 ; * \star \mathrm{p}<0.01 ;{ }^{* \star *} \mathrm{p}<0.001 ;+$ Marital status was excluded in 2019-20 due to multicollinearity 


\begin{tabular}{|c|c|c|c|c|c|c|}
\hline Characteristics & 2000 & 2005 & 2010 & 2014 & $2019-20$ & Pooled \\
\hline Low birth weight & $\begin{array}{l}1.28(0.56- \\
2.91)\end{array}$ & $\begin{array}{l}1.34 \\
(0.73- \\
2.46)\end{array}$ & $\begin{array}{l}0.80(0.55- \\
1.16)\end{array}$ & $\begin{array}{l}1.03(0.75- \\
1.41)\end{array}$ & $\begin{array}{l}0.98 \\
(0.72- \\
1.33)\end{array}$ & $\begin{array}{l}0.99 \\
(0.83- \\
1.20)\end{array}$ \\
\hline Big baby & $\begin{array}{l}2.00(1.11- \\
3.61)^{\star}\end{array}$ & $\begin{array}{l}1.42 \\
(0.96- \\
2.10)\end{array}$ & $\begin{array}{l}1.30(0.99- \\
1.71)\end{array}$ & $\begin{array}{l}1.57(1.22- \\
2.03)^{\star \star \star}\end{array}$ & $\begin{array}{l}1.84 \\
(1.34- \\
1.52)^{\star \star}\end{array}$ & $\begin{array}{l}1.45 \\
(1.26- \\
1.66)^{\star \star \star *}\end{array}$ \\
\hline \multicolumn{7}{|l|}{ Twin status } \\
\hline Singleton & 1 & 1 & 1 & 1 & 1 & 1 \\
\hline Multiple & $\begin{array}{l}11.07 \\
(3.36-36.5) \\
\star \star \star\end{array}$ & $\begin{array}{l}1.32 \\
(0.50- \\
3.50)\end{array}$ & $\begin{array}{l}6.08(3.20- \\
11.56)^{\star \star \star}\end{array}$ & $\begin{array}{l}6.43(3.88- \\
10.66)^{\star \star \star}\end{array}$ & $\begin{array}{l}3.63 \\
(2.04- \\
6.48)^{\star \star \star}\end{array}$ & $\begin{array}{l}4.77 \\
(3.50- \\
6.51)^{\star \star \star \star}\end{array}$ \\
\hline \multicolumn{7}{|l|}{ Region } \\
\hline Kigali City & 1 & 1 & 1 & 1 & 1 & 1 \\
\hline South & $\begin{array}{l}0.52(0.21- \\
1.29)\end{array}$ & $\begin{array}{l}0.38 \\
(0.18- \\
0.78)^{\star \star}\end{array}$ & $\begin{array}{l}1.04(0.49- \\
2.20)\end{array}$ & $\begin{array}{l}1.09(0.70- \\
1.69)\end{array}$ & $\begin{array}{l}1.00 \\
(0.68- \\
1.47)\end{array}$ & $\begin{array}{l}0.80 \\
(0.63- \\
1.02)\end{array}$ \\
\hline West & $\begin{array}{l}0.75(0.33- \\
1.71)\end{array}$ & $\begin{array}{l}0.53 \\
(0.26- \\
1.10)\end{array}$ & $\begin{array}{l}0.85(0.39- \\
1.86)\end{array}$ & $\begin{array}{l}0.94(0.62- \\
1.41)\end{array}$ & $\begin{array}{l}0.71 \\
(0.48- \\
1.04)\end{array}$ & $\begin{array}{l}0.70 \\
(0.55- \\
0.89)^{\star}\end{array}$ \\
\hline North & $\begin{array}{l}0.38(0.13- \\
1.12)\end{array}$ & $\begin{array}{l}0.19 \\
(0.08- \\
0.44)^{\star \star \star}\end{array}$ & $\begin{array}{l}0.70(0.30- \\
1.62)\end{array}$ & $\begin{array}{l}0.64(0.41- \\
1.02)\end{array}$ & $\begin{array}{l}0.61 \\
(0.39- \\
0.94)^{\star}\end{array}$ & $\begin{array}{l}050 \\
(0.38- \\
0.66)^{\star \star \star \star}\end{array}$ \\
\hline East & $\begin{array}{l}0.70(0.30- \\
1.63)\end{array}$ & $\begin{array}{l}0.35 \\
(0.16- \\
0.78)^{\star \star}\end{array}$ & $\begin{array}{l}0.92(0.42- \\
2.00)\end{array}$ & $\begin{array}{l}0.88(0.57- \\
1.34)\end{array}$ & $\begin{array}{l}0.72 \\
(0.49- \\
1.06)\end{array}$ & $\begin{array}{l}0.70 \\
(0.55- \\
0.89)^{\star}\end{array}$ \\
\hline \multicolumn{7}{|l|}{ Residence } \\
\hline Urban & 1 & 1 & 1 & 1 & 1 & 1 \\
\hline Rural & $\begin{array}{l}1.24(0.63- \\
2.42)\end{array}$ & $\begin{array}{l}1.19 \\
(0.67- \\
2.14)\end{array}$ & $\begin{array}{l}0.61(0.34- \\
1.10)\end{array}$ & $\begin{array}{l}0.87(0.60- \\
1.26)\end{array}$ & $\begin{array}{l}0.82 \\
(0.59- \\
1.14)\end{array}$ & $\begin{array}{l}0.91 \\
(0.74- \\
1.11)\end{array}$ \\
\hline \multicolumn{7}{|l|}{ Partners education } \\
\hline No formal & 1 & 1 & 1 & 1 & 1 & 1 \\
\hline Primary & $\begin{array}{l}0.46(0.23- \\
0.92)^{\star}\end{array}$ & $\begin{array}{l}1.62 \\
(0.95- \\
2.75)\end{array}$ & $\begin{array}{l}0.73(0.52- \\
1.03)\end{array}$ & $\begin{array}{l}0.97(0.72- \\
1.31)\end{array}$ & $\begin{array}{l}1.23 \\
(0.84- \\
1.80)\end{array}$ & $\begin{array}{l}0.96 \\
(0.80- \\
1.16)\end{array}$ \\
\hline $\begin{array}{l}\text { Secondary and } \\
\text { higher }\end{array}$ & $\begin{array}{l}0.35(0.14- \\
0.86)^{\star}\end{array}$ & $\begin{array}{l}0.83 \\
(0.44- \\
1.55)\end{array}$ & $\begin{array}{l}0.85(0.50- \\
1.45)\end{array}$ & $\begin{array}{l}1.09(0.74- \\
1.61)\end{array}$ & $\begin{array}{l}1.40 \\
(0.86- \\
2.25)\end{array}$ & $\begin{array}{l}0.95 \\
(0.75- \\
1.20)\end{array}$ \\
\hline
\end{tabular}




\section{Discussion}

Over the past two decades, the rate of CS in Rwanda significantly increased from 2.2-15.6\%, with disproportionately high rates among high socioeconomic groups and in urban areas, private health facilities and Kigali City. The rising trend in CS in Rwanda mirrors the global trend which shows an average annual increase of $4 \%$ [8]. The current CS rate of $15.6 \%$ in Rwanda is three times the rate in SSA, [10] and more than twice the average rate in Eastern and Southern African [34] though a previous study in Rwanda has shown a higher CS rate of $21 \%$ [35]. The increasing CS rate in Rwanda could indicate increased access to EmONC services and the high utilization of skilled delivery [36]. The current rate of CS is slightly above the 10-15\% WHO threshold for beneficial CS and likely to continue increasing if measures are not taken. Importantly, despite a 20\% decrease in the prevalence of CS in private hospitals between 2014 and 2019-20, there is a need to continue to monitor CS in private health facilities where there was a disproportionate increase in CS compared to public health facilities resulting in a four-fold difference in the prevalence of CS in 2019-20.

Overall, women of high socioeconomic status (education, occupation, and wealth) had increased odds of CS delivery, which reflects findings from previous studies [11, 37-40]. CS is a high-cost procedure in most settings making it accessible mostly to the rich. However, in Rwanda, a majority of the women are covered under the community-based health insurance schemes that have increased access to affordable healthcare by minimising the out-of-pocket expenditure and reducing the socioeconomic disparities in access and use of CS [19]. Moreover, women with high education levels are associated with ANC attendance [39, 41, 42], providing them with an opportunity to receive health education and counselling and early detection of risks. Mothers with high-risk pregnancies are also more likely to utilise ANC services than those with normal pregnancies though ANC attendance could also be indicative of better access to healthcare including CS.

Multiple pregnancies are high-risk and one of the obstetric indications for CS $[43,44]$. In our study, women with multiple pregnancies had higher odds of CS similar to previous studies [37, 45, 46]. In 2014, the rate of CS among women with multiple pregnancies was three times the average rate of CS in Rwanda, having doubled within the preceding five years. However, in 2019-20, the rate of CS among women with multiple pregnancies reduced by ten percentage points while that of singleton significantly increased $23.3 \%$. Hence, there is a need to review the indication for CS among women with multiple and singleton pregnancies to guide clinicians on women who are likely to benefit from the procedure and avoid unnecessary CS in this group.

Our study found lower odds of CS delivery among women who gave birth to female babies compared to those who delivered male babies similar to previous studies [47-52]. Male babies tend to be heavier than female babies [53-55] and are at an increased risk of adverse pregnancy outcomes [53]. We also found that women with two or more children had lower odds of CS delivery compared to primiparous women. This finding is consistent with previous studies in Kenya [56] and Ethiopia [46] and could be possibly attributed to poor dissolution of cervical collagen fibre in primigravid women due to their inexperience in labour [57]. Also, women who may have delivered their first baby via CS may have had successful vaginal delivery after a previous CS [58].

Big babies in the population-based analysis had lower odds of CS but had higher odds of CS in the hospitalbased analysis compared to normal-weight babies. Hospital-based studies have found a similar association between CS and big babies [59]. Large babies present difficulty in delivery possibly due to insufficient passage 
and prolonged labour. The lower odds of CS among big babies in the population-based analyses could be attributed to a delay in weighing of the babies delivered at home, who may have gained weight by the time of presenting at a health facility but also the fact that having a big baby is not an indication for CS and thus most big babies are delivered vaginally.

Our study adds to the evidence on CS in Rwanda by examining the population- and hospital-based trends and factors associated with CS in Rwanda over the past two decades using repeated nationally representative data making the findings generalisable to Rwanda. Our study, however, has some limitations that should be considered when interpreting the findings. First, there is a potential for overestimation or underestimation due to self-reporting of most variables and that the variables were measured at the time of the survey rather than at the time of delivery. Second, the dataset had missing observations and the study only included variables available from the dataset and could have missed important factors that predict CS in Rwanda. Finally, we could not assess the differences between the private and public health facilities due to the small sample of women who delivered in private health facilities.

\section{Conclusion}

Over the past 20 years, the rate of CS in Rwanda has seen an upward trend increasing seven-fold between 2000 and 2019-20 at health facility and population-level with persistent regional disparities over the years. The rates and odds of CS have been disproportionately high among women of high socioeconomic groups, and those who resided in urban areas and Kigali City, used private health facilities, had multiple pregnancies, and attended at least four antenatal care visits. Women in Rwanda seem to have increased access to and use of CS. However, the significant increase in the rate of CS is of concern due to the potential of unnecessary CS. Therefore, there is a need to examine the guidelines for CS use in Rwanda to ensure proper indications for use of CS are adopted for beneficial outcomes.

\section{Abbreviations}

ANC: Antenatal care; AOR: Adjusted odds ratio; BMI: Body mass index; Cl: Confidence Interval; COR: Crude odds ratio; CS: Caesarean section; DHS: Demographic Health Surveys; SSA: sub-Saharan Africa; SDGs: Sustainable development goals; WHO: World Health Organization

\section{Declarations}

\section{Ethical approval and consent to participate}

The study used anonymised secondary data from the RDHS, which received ethical approvals from the Rwanda National Ethics Committee and the Institutional Review Board of ICF International [20, 22-25]. All data collection procedures were performed in accordance with the declaration of Helsinki on protection of human subjects. We accessed the data upon approval of request to the DHS program and were used as per the data agreement.

\section{Consent for publication}




\section{Availability of data and materials}

The Rwanda Demographic Health Survey data used in this study is available on the Demographic Health Survey website at www.dhsprogram.com on request.

\section{Competing interests}

Authors declare no competing interests

\section{Funding}

This study did not receive any funding

\section{Authors' contributions}

PMK and LN conceptualised the study and obtained the data. CA, JO, GM and SG analysed the data and interpreted the results. All the authors reviewed the initial drafts and approved this final version.

\section{Acknowledgements}

We thank Measure DHS for granting us access to the data

\section{References}

1. World Health Organization: Trends in maternal mortality 2000 to 2017: estimates by WHO, UNICEF, UNFPA, World Bank Group and the United Nations Population Division. In. Geneva: World Health Organization; 2019: 104.

2. United Nations Inter-agency Group for Child Mortality Estimation (UN IGME): Levels \& Trends in Child Mortality: Report 2020 - Estimates developed by the UN Inter-agency Group for Child Mortality Estimation. In. New York, USA: United Nations Children's Fund; 2020.

3. Lawn JE, Blencowe H, Oza S, You D, Lee ACC, Waiswa P, Lalli M, Bhutta Z, Barros AJD, Christian P et al: Every Newborn: progress, priorities, and potential beyond survival. The Lancet 2014, 384(9938):189-205.

4. Bullough C, Meda N, Makowiecka K, Ronsmans C, Achadi EL, Hussein J: Current strategies for the reduction of maternal mortality. BJOG 2005, 112(9):1180-1188.

5. Campbell OM, Graham WJ, Lancet Maternal Survival Series Steering Group: Strategies for reducing maternal mortality: getting on with what works. The lancet 2006, 368(9543):1284-1299.

6. Betran AP, Torloni MR, Zhang JJ, Gulmezoglu AM, WHO Working Group on Caesarean Section: WHO Statement on Caesarean Section Rates. BJOG 2016, 123(5):667-670. 
7. Betran AP, Torloni MR, Zhang J, Ye J, Mikolajczyk R, Deneux-Tharaux C, Oladapo OT, Souza JP, Tuncalp O, Vogel JP et al: What is the optimal rate of caesarean section at population level? A systematic review of ecologic studies. Reprod Health 2015, 12:57.

8. Betrán AP, Ye J, Moller A-B, Zhang J, Gülmezoglu AM, Torloni MR: The increasing trend in caesarean section rates: global, regional and national estimates: 1990-2014. PloS one 2016, 11(2):e0148343.

9. Gibbons L, Belizan JM, Lauer JA, Betran AP, Merialdi M, Althabe F: Inequities in the use of cesarean section deliveries in the world. American journal of obstetrics and gynecology 2012, 206(4):331. e331-331. e319.

10. Betran AP, Ye J, Moller AB, Souza JP, Zhang J: Trends and projections of caesarean section rates: global and regional estimates. BMJ Glob Health 2021, 6(6).

11. Boatin AA, Schlotheuber A, Betran AP, Moller A-B, Barros AJ, Boerma T, Torloni MR, Victora CG, Hosseinpoor AR: Within country inequalities in caesarean section rates: observational study of 72 low and middle income countries. bmj 2018, 360.

12. Yaya S, Uthman OA, Amouzou A, Bishwajit G: Disparities in caesarean section prevalence and determinants across sub-Saharan Africa countries. Global health research and policy 2018, 3:19.

13. Lavender T, Hofmeyr GJ, Neilson JP, Kingdon C, Gyte GM: Caesarean section for non-medical reasons at term. Cochrane Database Syst Rev 2006(3):CD004660.

14. Nilsen C, Ostbye T, Daltveit AK, Mmbaga BT, Sandoy IF: Trends in and socio-demographic factors associated with caesarean section at a Tanzanian referral hospital, 2000 to 2013. Int J Equity Health 2014, 13(1):87.

15. Bayou YT, Mashalla YJS, Thupayagale-Tshweneagae G: Patterns of caesarean-section delivery in Addis Ababa, Ethiopia. African Journal of Primary Health Care \& Family Medicine 2016, 8(2):1-6.

16. World Health Organization: Appropriate technology for birth. Lancet 1985, 2:436-437.

17. Baradaran HR, Chu K, Cortier H, Maldonado F, Mashant T, Ford N, Trelles M: Cesarean Section Rates and Indications in Sub-Saharan Africa: A Multi-Country Study from Medecins sans Frontieres. PLoS One 2012, 7(9).

18. Odhiambo J, Ruhumuriza J, Nkurunziza T, Riviello R, Shrime M, Lin Y, Rusangwa C, Omondi JM, Toma G, Nyirimodoka A et al: Health Facility Cost of Cesarean Delivery at a Rural District Hospital in Rwanda Using Time-Driven Activity-Based Costing. Maternal and child health journal 2019, 23(5):613-622.

19. National Institute of Statistics of Rwanda (NISR): Rwanda Statistical Yearbook 2020. In. Kigali: National Institute of Statistics of Rwanda; 2020.

20. National Institute of Statistics of Rwanda, Finance Mo, Economic Planning/Rwanda, Ministry of Health/Rwanda, ICF International: Rwanda Demographic and Health Survey 2014-15. In. Kigali, Rwanda: National Institute of Statistics of Rwanda, Ministry of Finance and Economic Planning/Rwanda, Ministry of Health/Rwanda, and ICF International; 2016.

21. The World Bank: Macro Poverty Outlook Country-by-country Analysis and Projections for the Developing World Spring Meetings 2021. In. Washington DC: International Bank for Reconstruction and Development / The World Bank; 2021.

22. Institut National de la Statistique du Rwanda - INSR, ORC Macro: Rwanda Demographic and Health Survey 2005. In. Calverton, Maryland, USA: INSR and ORC Macro; 2006. 
23. National Institute of Statistics of Rwanda - NISR, Ministry of Health - MOH/Rwanda, ICF International: Rwanda Demographic and Health Survey 2010. In. Calverton, Maryland, USA: NISR/Rwanda, MOH/Rwanda, and ICF International; 2012.

24. Office National de la Population - ONAPO/Rwanda, ORC Macro: Rwanda Enqulte Démographique et de Santé 2000. In. Kigali, Rwanda: Ministére de la Santé, Office National de la Population and ORC Macro; 2001.

25. National Institute of Statistics of Rwanda, Ministry of Health Rwanda, ICF International: Rwanda Demographic and Health Survey 2019-20 Final Report. In. Kigali, Rwanda and Rockville, Maryland, USA: National Institute of Statistics of Rwanda and ICF International; 2021: 619.

26. Wu JM, Viswanathan M, Ivy JS, North Carolina Research Collaborative on Mode of D: A conceptual framework for future research on mode of delivery. Maternal and child health journal 2012, 16(7):14471454.

27. Jadoon B, Mahaini R, Gholbzouri K: Determinants of over and underuse of caesarean births in the Eastern Mediterranean Region: an updated review. East Mediterr Health J 2019, 25(11):837-846.

28. Azami-Aghdash S, Ghojazadeh M, Dehdilani N, Mohammadi M, Asl Amin Abad R: Prevalence and Causes of Cesarean Section in Iran: Systematic Review and Meta-Analysis. Iran J Public Health 2014, 43(5):545555.

29. Gebreegziabher Hailu A, Kebede Fanta T, Tekulu Welay F, Etsay Assefa N, Aregawi Hadera S, Aregawi Gebremeskel G, Weldeslassie Gebremedhin H, Asefa GG: Determinants of Cesarean Section Deliveries in Public Hospitals of Addis Ababa, Ethiopia, 2018/19: A Case-Control Study. Obstet Gynecol Int 2020, 2020:9018747.

30. Hernandez-Vasquez A, Chacon-Torrico H, Bendezu-Quispe G: Differences in the prevalence of cesarean section and associated factors in private and public healthcare systems in Peru. Sexual \& reproductive healthcare : official journal of the Swedish Association of Midwives 2020, 26:100570.

31. Karim F, Ali NB, Khan ANS, Hassan A, Hasan MM, Hoque DME, Billah SM, El Arifeen S, Chowdhury MAK: Prevalence and factors associated with caesarean section in four Hard-to-Reach areas of Bangladesh: Findings from a cross-sectional survey. PLoS One 2020, 15(6):e0234249.

32. Nilsen C, Ostbye T, Daltveit AK, Mmbaga BT, Sandoy IF: Trends in and socio-demographic factors associated with caesarean section at a Tanzanian referral hospital, 2000 to 2013. Int J Equity Health 2014, 13:87.

33. Elm Ev, Altman DG, Egger M, Pocock SJ, Gøtzsche PC, Vandenbroucke JP: Strengthening the reporting of observational studies in epidemiology (STROBE) statement: guidelines for reporting observational studies. BMJ 2007, 335(7624):806-808.

34. Boerma T, Ronsmans C, Melesse DY, Barros AJD, Barros FC, Juan L, Moller AB, Say L, Hosseinpoor AR, Yi $\mathrm{M}$ et al: Global epidemiology of use of and disparities in caesarean sections. Lancet 2018, 392(10155):1341-1348.

35. Kakoma J-B: Cesarean section indications and anthropometric parameters in Rwandan nulliparae: preliminary results from a longitudinal survey. The Pan African Medical Journal2016, 24.

36. Gurusamy PSR, Janagaraj PD: A Success Story: The Burden of Maternal, Neonatal and Childhood Mortality in Rwanda - Critical Appraisal of Interventions and Recommendations for the Future. Afr J Reprod 
Health 2018, 22(2):9-16.

37. Adewuyi EO, Auta A, Khanal V, Tapshak SJ, Zhao Y: Cesarean delivery in Nigeria: prevalence and associated factors-a population-based cross-sectional study. BMJ Open 2019, 9(6):e027273.

38. Cavallaro FL, Cresswell JA, Franca GV, Victora CG, Barros AJ, Ronsmans C: Trends in caesarean delivery by country and wealth quintile: cross-sectional surveys in southern Asia and sub-Saharan Africa. Bull World Health Organ 2013, 91(12):914-922D.

39. Leone T, Padmadas SS, Matthews Z: Community factors affecting rising caesarean section rates in developing countries: an analysis of six countries. Soc Sci Med 2008, 67(8):1236-1246.

40. Taye MG, Nega F, Belay MH, Kibret S, Fentie Y, Addis WD, Fenta E: Prevalence and factors associated with caesarean section in a comprehensive specialized hospital of Ethiopia: A cross-sectional study; 2020. Ann Med Surg (Lond) 2021, 67:102520.

41. Nambile Cumber S: Importance of Antenatal Care Services to Pregnant Women at the Buea Regional Hospital Cameroon. Journal of Family Medicine and Health Care 2016, 2(4):23-29.

42. Chigbu CO, Iloabachie GC: The burden of caesarean section refusal in a developing country setting. BJOG 2007, 114(10):1261-1265.

43. Robson MS: Classification of caesarean sections. Fetal Matern Med Rev 2001, 12(1):23-39.

44. Robson MS: Use of indications to identify appropriate caesarean section rates. The Lancet Global Health 2018, 6(8):e820-e821.

45. Akinola OI, Fabamwo AO, Tayo AO, Rabiu KA, Oshodi YA, Alokha ME: Caesarean section-an appraisal of some predictive factors in Lagos Nigeria. BMC Pregnancy Childbirth 2014, 14(1):217.

46. Yisma E, Smithers LG, Lynch JW, Mol BW: Cesarean section in Ethiopia: prevalence and sociodemographic characteristics. J Matern Fetal Neonatal Med 2019, 32(7):1130-1135.

47. Antonakou A: The Effect of Fetal Gender on the Delivery Outcome in Primigravidae Women with Induced Labours for all Indications. Journal of Clinical and Diagnostic Research 2016.

48. Bekedam DJ, Engelsbel S, Mol BW, Buitendijk SE, van der Pal-de Bruin KM: Male predominance in fetal distress during labor. Am J Obstet Gynecol 2002, 187(6):1605-1607.

49. Khalil MM, Alzahra E: Fetal gender and pregnancy outcomes in Libya: a retrospective study. Libyan J Med 2013, 8(1).

50. Liu Y, Li G, Zhang W: Effect of fetal gender on pregnancy outcomes in Northern China. J Matern Fetal Neonatal Med 2017, 30(7):858-863.

51. Schildberger B, Leitner H: Foetal Gender and Obstetric Outcome. Geburtshilfe Frauenheilkd 2016, 76(3):255-260.

52. Weissmann-Brenner A, Simchen MJ, Zilberberg E, Kalter A, Dulitzky M: Combined effect of fetal sex and advanced maternal age on pregnancy outcomes. Med Sci Monit 2015, 21:1124-1130.

53. Di Renzo GC, Rosati A, Sarti RD, Cruciani L, Cutuli AM: Does fetal sex affect pregnancy outcome? Gend Med 2007, 4(1):19-30.

54. Dunn L, Prior T, Greer R, Kumar S: Gender specific intrapartum and neonatal outcomes for term babies. Eur J Obstet Gynecol Reprod Biol 2015, 185:19-22. 
55. Sheiner E, Levy A, Katz M, Hershkovitz R, Leron E, Mazor M: Gender Does Matter in Perinatal Medicine. Fetal Diagn Ther 2004, 19(4):366-369.

56. van der Spek L, Sanglier S, Mabeya HM, van den Akker T, Mertens PLJM, Houweling TAJ: Socioeconomic differences in caesarean section - are they explained by medical need? An analysis of patient record data of a large Kenyan hospital. International Journal for Equity in Health 2020, 19(1):117-117.

57. Vrouenraets FP, Roumen FJ, Dehing CJ, van den Akker ES, Aarts MJ, Scheve EJ: Bishop score and risk of cesarean delivery after induction of labor in nulliparous women. Obstet Gyneco/2005, 105(4):690-697.

58. Kalisa R, Rulisa S, van Roosmalen J, van den Akker T: Maternal and perinatal outcome after previous caesarean section in rural Rwanda. BMC Pregnancy Childbirth 2017, 17(1):272.

59. Tarimo CS, Mahande MJ, Obure J: Prevalence and risk factors for caesarean delivery following labor induction at a tertiary hospital in North Tanzania: a retrospective cohort study (2000-2015). BMC Pregnancy Childbirth 2020, 20(1):173.

\section{Supplementary Files}

This is a list of supplementary files associated with this preprint. Click to download.

- Supplementarymaterials.docx 